\title{
ENTIRE FUNCTIONS, IN THE CLASSIFICATION OF DIFFERENTIABLE GERMS TANGENT TO THE IDENTITY, IN ONE OR TWO VARIABLES
}

\author{
PATRICK AHERN AND JEAN-PIERRE ROSAY
}

\begin{abstract}
This paper presents a survey and some (hopefully) new facts on germs of maps tangent to the identity (in $\mathbb{R}, \mathbb{C}$, or $\mathbb{R}^{2}$ ), (maps $f$ defined near 0 , such that $f(0)=0$, and $f^{\prime}(0)$ is the identity). Proofs are mostly original.

The paper is mostly oriented towards precise examples and the questions of descriptions of members in the conjugacy class, flows, $k$ th root.

It happened that entire functions provide clear and easy examples. However they should be considered just as a tool, not as the main topic. For example in Proposition 2 the function $z \mapsto z+z^{2}$ should be better thought of as the map $(x, y) \rightarrow\left(x+x^{2}-y^{2}, y+2 x y\right)$.
\end{abstract}

As original results we have

Proposition 1. Let $f(z)=z+\sum_{j=2}^{\infty} a_{j} z^{j}$. Assume that $f$ is the time $t$ map of a smooth vector field $X$ defined in a neighborhood of 0 in $\mathbb{R}^{2}, X(0)=0$. If $f$ is entire then $f(z) \equiv z$. If $f$ is meromorphic on $\mathbb{C}$ (or more generally has a countable set of singularities in $\mathbb{C}$ ) then $f(z)=\frac{z}{1-\alpha z}$ for some $\alpha \in \mathbb{C}$.

In $\S$ VIII a more precise version is given, and "smoothness" is made precise. As usual $\mathbb{C}$ and $\mathbb{R}^{2}$ are identified, when convenient.

Proposition 2. The only germs of entire functions $G(z)=z+\cdots$ which are $\mathscr{C}^{6}$ conjugate to $z+z^{2}$ in a neighborhood of 0 in $\mathbb{C}$ are the functions $G(z)=$ $z+a z^{2}$, for $a \neq 0$. In no neighborhood of 0 does the map $z+z^{2}$ have a kth root $(k>1)$ of class $\mathscr{C}^{6}$ (i.e. a $\mathscr{C}^{6}$ diffeomorphism $f$ of $\left(\mathbb{R}^{2}, 0\right)$ such that $\left.f \circ \cdots \circ f=z+z^{2}\right)$.

Notice that, as explained below, it follows from a work by Takens that $x+x^{2}$ has a (unique) smooth square root, near 0 , on $\mathbb{R}$.

Proposition 1 and the second half of Proposition 2 have been obtained, in the holomorphic setting, by G. Szekeres [22] and I. N. Baker [1]-[4] (see also [22]), in papers which deserve to be much better known. In the ignorance of these papers, we developed a different approach. After studying the vector fields $z^{2}+C z^{3}$ (or $z^{p}+C z^{2 p-1}$ ), we can give a short and transparent (we do not claim better) proof of Baker's and Szekeres's theorems. It is worthwhile to

Received by the editors November 23, 1993 and, in revised form, March 25, 1994. $26 \mathrm{E} 10$.

1991 Mathematics Subject Classification. Primary 30D05, 34A20; Secondary 26A18, 26E05,

Partially supported by a National Science Foundation grant. 
notice that one case ( $\S \mathrm{IV})$ is really immediate. The smooth case is later deduced from the analytic case. Proposition 6 in $\S$ VIII, and its corollary, provide the tools for applying the analytic results in the differentiable setting (conjugators and roots of holomorphic functions must be holomorphic or antiholomorphic). Proposition 2 is given as an illustration, to illustrate how discrete and no longer continuous dynamics can be used. Monodromy questions are more difficult to handle, as will be explained.

As already said, this paper is also intended as a partial survey. And we include some description of works by Fatou, Sternberg, Takens, Ecalle, and Voronin, but the reader should also look at [21]. We even remark, in $\S I$, why the case of maps tangent to the identity is of special interest. We will cover the formal, smooth, and analytic theories, which are strikingly different. In one real variable, the formal and the $\mathscr{C}^{\infty}$ classification agree, for germs which are tangent, but not infinitely tangent to the identity (Takens), but they differ from the analytic classification (Ecalle-Voronin). In two real variables all these theories differ. The plan of this paper is as follows.

I The "basic scheme" for conjugacy. Preliminaries

II (1) Formal theory in one variable

(2) Classification of holomorphic or meromorphic vector fields

III Takens's $\mathscr{C}^{\infty}$ classification (one variable), and Robbin's classification

IV First, easy, examples

V Baker's and Szekeres's theorems on flows

VI The function $z+z^{2}$

VII Analytic classification in one variable by Ecalle and Voronin. Sketch of Voronin's theory

VIII Two real variables

Definitions. Let $X$ be a $\mathscr{C}^{l}$ vector field defined near 0 , in $\mathbb{R}$ or $\mathbb{R}^{2}$. Assume that $X(0)=0$. Then for every $T>0$, there exists $r>0$ so that the differential equation $\dot{R}(t)=X(R(t))$ can be solved for time $t \in[-T,+T]$, with arbitrary initial data $R(0)=R_{0},\left|R_{0}\right|<r$. Upper dot denotes differentiation with respect to time. The map $R_{0} \rightarrow R(t)$ is the time $t$ map of the flow (defined on a neighborhood of 0 which shrinks as $|t| \rightarrow+\infty$ ). We denote the (germ of the) map $T_{t}^{X}$ (or $T_{t}$ if there is no ambiguity). In the case of a holomorphic vector field $X(z)=\sum_{j=1}^{\infty} b_{j} z^{j}$ defined near 0 in $\mathbb{C}$, the corresponding time $t$ maps are holomorphic:

$$
T_{t}^{X}(z)=e^{b_{1} t} z+\sum_{j=2}^{\infty} B_{j}(t) z^{j}
$$

(see $\S$ II for a computation of the $B_{j}$ 's).

Two germs of map $f$ and $\tilde{f}$ at 0 (both from $(\mathbb{R}, 0)$ into $(\mathbb{R}, 0)$ or from $\left(\mathbb{R}^{2}, 0\right)$ into $\left.\left(\mathbb{R}^{2}, 0\right)\right)$, vanishing at 0 are conjugate to each other if and only if there exists a local change of variable $\varphi$ at $0, \varphi(0)=0$, such that $f=$ $\varphi \circ \tilde{f} \circ \varphi^{-1}$, or equivalently $f \circ \varphi=\varphi \circ \tilde{f}$.

We may be looking for such changes of variables either formal, or analytic, or $\mathscr{C}^{l}$ (we then say that $f$ and $\tilde{f}$ are $\mathscr{C}^{l}$ conjugate).

If $f$ is a map, and $k$ is a positive integer $f^{(k)}$ denotes the $k$-fold product $f \circ f \circ \cdots \circ f$. For $k<0, f^{(k)}=\left[f^{(-k)}\right]^{-1}$, but $f^{-1}$ will be used instead of $f^{(-1)}$. 
Acknowledgments. We wish to express our gratitude to our colleague Joel Robbin, for his very generous help. The final part of this paper has been written while the second author was visiting the University of Grenoble. He wishes to thank this institution, and he wishes to thank especially A. Dufresnoy and F. Sergeraert.

\section{BASIC SCHEME}

1. By basic scheme we refer to the following simple trick. If $F$ and $G$ are maps or germs of a map so that $\lim G^{(-n)} \circ F^{(n)}$ exists, then obviously

$$
G^{-1}\left(\lim G^{(-n)} \circ F^{(n)}\right) \circ F=\lim G^{(-n)} F^{(n)} .
$$

So if

$$
\psi=\lim G^{(-n)} \circ F^{(n)}
$$

we have $G^{-1} \circ \psi \circ F=\psi$. Equivalently: $\psi \circ F=G \circ \psi$, or assuming $\psi$ (locally) invertible $F=\psi^{-1} \circ G \circ \psi$.

The basic scheme was used by Fatou in 1919 for the sector conjugacy used (and proved differently) by Voronin. But we begin with the simpler problem of linearization (following Sternberg), so that the reader will get used to the trick.

2. Linearization of contractions. See [19] and [20]. For the reader, we just illustrate the trick on the following example:

Let $F(z)=a z+\sum_{j=2}^{n} a_{j} z^{j}$ be a germ of a holomorphic map defined near 0 in $\mathbb{C}$. Assume $|a|<1$. Fix $A$ so that $A^{2}<|a|<A$. Set $G(z)=a z$, a linear map. There exists $r>0$ so that for $|z|<r,|F(z)|<A|z|$. Then we have $G^{(-n)} \circ F^{(n)}(z)=a^{-n} F^{(n)}(z)$, so

$$
\begin{aligned}
\left(G^{(-n-1)} \circ F^{(n+1)}-G^{(-n)} \circ F^{(n)}\right)(z) & =a^{-n}\left(a^{-1} F\left(F^{(n)}(z)\right)-F^{(n)}(z)\right) \\
& =a^{-n} \mathscr{O}\left(A^{2 n}|z|\right)
\end{aligned}
$$

(using $a^{-1} F(\zeta)-\zeta=\mathscr{O}\left(|\zeta|^{2}\right)$ ). Finally $G^{(-n-1)} \circ F^{(n+1)}-G^{(-n)} \circ F^{(n)}(z)=$ $\left(\frac{A^{2}}{a}\right)^{n} \mathscr{O}(1)$. This proves uniform convergence of $G^{(-n)} \circ F^{(n)}$ on $|z|<r$, and since $\left(G^{(-n)} \circ F^{(n)}\right)^{\prime}(0)=1$, the limit $\psi$ satisfies also $\psi^{\prime}(0)=1$.

In fact $\psi$ converges on the basin of attraction of 0 for $F$ (replacing $z$ above by $F^{(k)}(z)$ for some large $\left.k\right)$.

In several variables as pointed out in [20] this scheme works only if the eigenvalues are roughly speaking of the same size (square of largest one has to be smaller than the smallest one: in order that the linear part absorbs the quadratic part). See also [15], where this is used in the construction of FatouBieberbach regions.

Sometimes the basic scheme applies (only) after minor modifications ([7, 20, 15]).

3. The linearization of $F(z)=a z+\sum_{j=2}^{\infty} a_{j} z^{j}$ for $|a|>1$ is achieved by linearizing $F^{-1}$. The case $|a|=1$ is the object of a famous work by Siegel [18]. If $a=1$, linearization is simply impossible unless $F(z) \equiv z$. This is why we concentrate on this class (which is the object of the theories of Ecalle and Voronin). Siegel's work, like earlier work by Poincaré, is based on estimating coefficients in formal power series. 
Once a map is linearized the questions of flow and $k$ th roots are trivially answered.

II

Here we collect facts which have been used by many authors. Verifications for the formal theory are totally trivial, and are mostly left to the reader.

\section{Formal theory.}

1.1. Conjugacy class. As for convergent series two formal power series $f=$ $z+\sum_{j=2}^{\infty} a_{j} z^{j}$ and $\tilde{f}=z+\sum_{j=2}^{\infty} \tilde{a}_{j} z^{j}$ are said to be conjugate if and only if there exists a formal power series $\varphi=\sum_{j=1}^{\infty} \varphi_{j} z^{j}, \varphi_{1} \neq 0$, so that $f \circ \varphi=\varphi \circ \tilde{f}$ (as formal power series).

The following is known:

(i) If $a_{2} \neq 0, \tilde{f}$ is conjugate to $f$ if and only if $\tilde{a}_{2} \neq 0$ and $a_{3} / a_{2}^{2}=\tilde{a}_{3} / \tilde{a}_{2}^{2}$.

(ii) There are a unique $p \geq 0$ and a unique $\lambda \in \mathbb{C}$, so that $f$ is (formally) conjugate to $z+z^{p}+\lambda z^{2 p-1}$. And $p$ is the smallest integer $\geq 2$ so that $a_{p} \neq 0$.

1.2. Flows. Given a formal vector field $X=\sum_{j=1}^{\infty} b_{j} z^{j}$ it generates a formal flow $T_{t}^{X}(z)=\sum_{j=1}^{\infty} a_{j}(t) z^{j}$ defined by $T_{0}^{X}(z)=z, \dot{T}_{t}^{X}(z)=X\left(T_{t}^{X}(z)\right)$, as formal power series in $z$.

The formal flow of a formal power series exists and is unique.

Indeed,

$$
\begin{aligned}
\dot{T}_{t}(z) & =\sum \dot{a}_{j}(t) z^{j}=X\left(T_{t}(z)\right) \\
& =b_{1}\left(a_{1} z+a_{2} z^{2}+\cdots\right)+b_{2}\left(a_{1} z+a_{2} z^{2}+\cdots\right)^{2}+b_{3}()^{3}+\cdots .
\end{aligned}
$$

This gives $\dot{a}_{1}=a_{1} b_{1}, \dot{a}_{2}=b_{1} a_{2}+b_{2} a_{1}^{2}, \dot{a}_{3}=b_{1} a_{3}+2 b_{2} a_{1} a_{2}+b_{3} a_{1}^{3}, \ldots$ The first equation gives $a_{1}(t)=e^{b_{1} t}$ since $a_{1}(0)=1$; the remaining equations are then solved inductively for $a_{j}$, using the initial condition $a_{j}(0)=0, j \geq 2$.

One has the following commutation relation:

$$
X\left(T_{t}(z)\right)=X(z) T_{t}^{\prime}(z)
$$

(prime denoting formal differentiation with respect to $z$ ), that one obtains by differentiating at $t=t_{0}$

$$
T_{t}(z)=T_{t_{0}}\left[T_{t-t_{0}}(z)\right]
$$

The following will be used later:

Lemma 1. Suppose that $X(z)=b_{p} z^{p}+O\left(z^{p+1}\right), b_{p} \neq 0$, is a formal field and that for some $t$ its formal flow has the form $T_{t}(z)=z+a_{q} z^{q}+O\left(z^{q+1}\right), a_{q} \neq 0$, $q \geq 2$. Then $p=q$.

Proof. We apply the commutation equation:

$b_{p}\left(z+a_{q} z^{q}+\cdots\right)^{p}+b_{p+1}\left(z+a_{q} z^{q}+\cdots\right)^{p+1}+\cdot=\left(b_{p} z^{p}+\cdots\right)\left(1+q a_{q} z^{q-1}+\cdots\right)$.

We equate the coefficients of $z^{p+q-1}$; on the left-hand side it is $b_{p+q-1}+p b_{p} a_{q}$; on the right-hand side it is $b_{p+q-1}+q b_{p} a_{q}$. Since $b_{p} a_{q} \neq 0$, we have $p=q$.

Corollary. If for some value of $t, a_{1}(t)=1$, but $T_{t}(z) \not \equiv z$, then $b_{1}=0$ and $a_{1}(t) \equiv 1$ for all $t$.

Proof. The lemma tells us that $b_{1}=0$, but then $a_{1}=e^{b_{1} t} \equiv 1$. 
1.3. Square roots. Given a formal power series $f=z+\sum_{j=2}^{\infty} a_{j} z^{j}$ there is a unique formal power series $g=z+\sum_{j=2}^{\infty} \alpha_{j} z^{j}$ so that $g \circ g=f$.

Proof. Immediate. Indeed $g \circ g=f$ gives

$$
\left(z+\sum_{j=2}^{\infty} \alpha_{j} z^{j}\right)+\sum_{j=2}^{\infty} \alpha_{j}\left(z+\sum_{k=2}^{\infty} \alpha_{k} z^{k}\right)^{j}=z+\sum_{j=2}^{\infty} a_{j} z^{j} .
$$

For $j \geq 2$ the coefficient of $z^{j}$ in the left-hand side is $2 \alpha_{j}+R_{j}$ where $R_{j}$ depends only on $\alpha_{2}, \ldots, \alpha_{j-1}$. So the $\alpha_{j}$ 's are successively uniquely determined.

The results explained so far in this section show that for the questions studied in this paper (square roots, flows, conjugacy), there are no, or few, restrictions at the level of jets. The whole problem is a question of convergence.

2. Normal forms of vector fields (one variable). The theory can be done at the level of formal power series, or in the $\mathscr{C}^{\infty}$ category [23] or in the holomorphic setting. Surprisingly (or not) the classifications agree. Here we will provide all details for the case of vector fields defined near 0 in $\mathbb{C}$. It will be then convenient to allow a singularity (pole) at 0 .

Definition. Suppose $X, \tilde{X}$ are holomorphic vector fields (in coordinates, simply two holomorphic functions), defined in some deleted neighborhood of 0 in $\mathbb{C}$. We say $X$ and $\widetilde{X}$ are locally equivalent if there is a one-to-one holomorphic map $\varphi$ defined in a neighborhood of 0 with $\varphi(0)=0$ so that $\varphi_{*}(X)=\tilde{X}$, in some deleted neighborhood of 0 .

Note that if $X$ is locally equivalent to $\tilde{X}$ and $X$ has a pole of order $p$ at 0 so does $\tilde{X}$. Similarly if $X$ is holomorphic at 0 and vanishes to order $p$, the same is true for $\widetilde{X}$.

The following proposition is NOT original. But its proof is included because of the contrast with the (only) formal theory of flows or square roots.

Proposition 3. Suppose $X$ is a holomorphic vector field defined in a deleted neighborhood of 0 .

(i) If $X$ is holomorphic at 0 and vanishes to order exactly $p \geq 2$, then $X$ is equivalent to $X_{0}(z)=z^{p}+\lambda z^{2 p-1}$, for a unique $\lambda \in \mathbb{C}$.

(ii) If $X$ is holomorphic at $0, X(0)=0$, and $X^{\prime}(0)=b \neq 0$ then $X$ is equivalent to $X_{0}(z)=b z$.

(iii) If $X$ has a pole of order $p \geq 0$ then $X$ is equivalent to $X_{0}(z)=z^{-p}$.

(Here by pole of order 0 we mean $X$ is holomorphic at 0 and $X(0) \neq 0$.)

Proof. (i) We first claim that it is easy to find a local change of variable $\psi$ and a vector field $\widetilde{X}=z^{p}+\lambda z^{2 p-1}+\sum_{j=2 p}^{\infty} \tilde{b}_{j} z^{j}$ so that $\tilde{X}=\psi_{*}(x)$. It is convenient to do this in several steps. First, let $\psi^{0}$ be the homothety $\psi^{0}(z)=\mu z$, with $\mu^{p-1}=b_{p}$. Then $\psi_{0}^{*}(X)=z^{p}+\cdots$. Next assume that for some $r, 0<r<$ $p-1$, we have already found $\psi_{r}$ so that $\psi_{r}(X)=z^{p}+c_{p+r} z^{p+r}+\cdots$. Set $\tau(z)=z-A z^{r+1}$. 
Then

$$
\begin{aligned}
\tau_{*}^{-1}\left(\psi_{r}(X)\right)(z)= & \frac{\psi_{r}(X)(\tau(z))}{\tau^{\prime}(z)} \\
= & \frac{\left(z-A z^{r+1}\right)^{p}+c_{p+r}\left(z-A z^{r+1}\right)^{p+r}+\cdots}{\left(1-(r+1) A z^{r}\right)} . \\
\tau_{*}^{-1} \psi_{r}(X)= & {\left[\left(z^{p}-p A z^{p+r}+\cdots\right)+\left(c_{p+r} z^{p+r}+\cdots\right)\right] } \\
& \cdot\left(1+(r+1) A z^{r}+(r+1)^{2} A^{2} z^{2 r}+\cdots\right) \\
= & z^{p}+\left[A(-p+r+1)+c_{p+r}\right] z^{p+r} \\
& + \text { higher-order terms. }
\end{aligned}
$$

Since $-p+r+1 \neq 0$ (i.e. $p+r \neq 2 p-1$ ) we can choose $A=\frac{-c_{p+r}}{-p+r+1}$. And then

$$
\left(\tau^{-1} \psi_{r}\right)_{*}(X)=z^{p}+c_{p+r+1}^{\prime} z^{p+r+1}+\cdots .
$$

In finitely many steps we get $\widetilde{X}=\psi_{*}(X)$ as desired.

We leave to the reader to extract from the above computations the proof of the uniqueness of $\lambda$. Now, we have to explain how to make a (convergent) change of variable $\rho$ so that $\rho_{*}\left(z^{p}+\lambda z^{2 p-1}\right)=\widetilde{X}$, which means

$$
\left(z^{p}+\lambda z^{2 p-1}\right) \rho^{\prime}(z)=\tilde{X}(\rho(z)) .
$$

Set $\rho(z)=z+z^{p} h$, where $h$ is to be found. Then $(*)$ is

$$
\left(z^{p}+\lambda z^{2 p-1}\right)\left(1+p z^{p-1} h+z^{p} h^{\prime}\right)=\widetilde{X}\left(z+z^{p} h\right) ;
$$

i.e.

$$
\left(1+\lambda z^{p-1}\right) h^{\prime}(z)=\frac{1}{z^{2 p}}\left[\begin{array}{c}
\tilde{X}\left(z+z^{p} h\right)-z^{p}-(\lambda+p h) z^{2 p-1} \\
-\lambda p h z^{3 p-2}
\end{array}\right]
$$

All what we have to prove is that the right-hand side is a holomorphic function of the two variables $(z, h)$ is some neighborhood of 0 .

Then $h$ is obtained by solving the differential equation. Remember $\widetilde{X}=$ $z^{p}+\lambda z^{2 p-1}+$ higher-order terms. So

$$
\begin{aligned}
\widetilde{X}\left(z+z^{p} h\right) & =\left(z+z^{p} h\right)^{p}+\lambda\left(z+z^{p} h\right)^{2 p-1}+\cdots \\
& =z^{p}+(\lambda+p h) z^{2 p-1}+\lambda p h z^{3 p-2}+R(z, h),
\end{aligned}
$$

where $R(z, h)$ is a convergent power series of $(z, h)$ (in some "small" neighborhood of $(0,0)$ ), with no term of degree less than $2 p$ in $z$. So the right-hand side in $(* *)$ reduces to $\frac{R(z, h)}{z^{2 p}}$, which is indeed holomorphic near 0 , and this ends the proof of $i$.

(ii) is proved in the same way as (i). It is in fact easier. It is stated separately just because when $p=1,2 p-1=1$ as well.

(iii) This is the easiest case. We want to find a holomorphic map $\varphi$ with $\varphi(0)=0$ and $\varphi^{\prime}(0) \neq 0$ so that

$$
X(z) \varphi^{\prime}(z)=\varphi(z)^{-p}
$$

that is, $\varphi(z)^{p} \varphi^{\prime}(z)=\frac{1}{X(z)}$. But $\frac{1}{X(z)}$ has a zero of order $p$ at 0 and hence has a primitive of the form $z^{p+1} h(z)$ where $h(0) \neq 0$. Take $\varphi(z)=z g(z)$ where $g$ is a $(p+1)$ th root of $(p+1)^{(p+1)} h$. 


\section{TAKENS'S CLASSIFICATION}

This section deals with $\mathscr{C}^{\infty}$ diffeomorphisms in $\mathbb{R}$.

1. Theorem 2 in [23] says in particular the following:

Theorem (Takens). Let $\chi:(\mathbb{R}, 0) \rightarrow(\mathbb{R}, 0)$ be a $\mathscr{C}^{\infty}$ diffeomorphism such that $\chi(x)=x+x^{p} F(x)$ with $F(0) \neq 0$ and $p \geq 2$. Then there is a $\mathscr{C}^{\infty}$ diffeomorphism $\varphi:(\mathbb{R}, 0) \rightarrow(\mathbb{R}, 0)$ such that in some neighborhood of $0 \in \mathbb{R}$, $\varphi \circ \chi \circ \varphi^{-1}=x+\delta x^{p}+\alpha x^{2 p-1}$. If $k$ is even $\delta=1$, if $k$ is odd $\delta= \pm 1$, and $\alpha$ is entirely determined by the $(2 p-1)$ jet of $\chi$ at 0 .

The proof given in [23] is elegant. But we wish to show that the theorem can also be obtained by applying the basic scheme explained in $\S I$ (may be painful, but straightforward!) We will need the following lemma proved in $\S 4$ below. This may be a well-known result, and it is not intended to be sharp.

Lemma 2. Let $\chi$ be a $\mathscr{C} \infty$ diffeomorphism of $[0,1]$ onto itself. Assume that $\chi(0)=0, \chi(1)=1, \chi^{\prime}(0)=1, \chi^{\prime}(1)=1, \chi(x) \neq x$ for all $x \in(0,1)$. And assume that $x-\chi(x)$ is not flat at 0 nor at 1 . Then for every $k \in \mathbb{N}$, there exists $s$ so that

$$
\left\|\chi^{(n)}\right\|_{\mathscr{C}_{k}}=\mathscr{O}\left(n^{s}\right) \quad \text { as }|n| \rightarrow \infty(n \in Z) .
$$

Nonflat means that some derivative does not vanish. It is enough to consider the case $\chi(x)<x$ on $(0,1)$, and let $n$ tend to $+\infty$. Then $[0,1)$ is the basin of attraction of 0 for forward iteration $\left(\chi^{(n)}, n>0\right)$, while $(0,1]$ is the basin of attraction of 1 for backward iteration $(n<0)$. The situation is simply reversed if $\chi(x)>x$, or $n$ tends to $-\infty$ (or, replace $\chi$ by the conjugate map $\left.\chi_{1}(x)=1-\chi(1-x)\right)$. We postpone the proof of Lemma 2 .

Proof of Theorem. Let $\chi$ be as in the statement of the theorem. Using the formal theory and the fact that any formal power series can be matched by the Taylor expansion of some $\mathscr{C}^{\infty}$ function (an old result by Borel) we can assume without loss of generality that $\chi$ and $x+\delta x^{p}+\alpha x^{2 p-1}$ match to infinite order at 0 , with $\delta= \pm 1$. In fact replacing $\chi$ by $\chi^{-1}$ if needed we can assume $\delta=-1$ (a slight change of notation, with respect to the statement of the theorem).

Although a posteriori this is useless, we are going to modify our functions $\chi$ and $x-x^{p}+\alpha x^{2 p-1}$ off some neighborhood of 0 . And for the present time we restrict our attention to the interval $[0,1]$. Take $\chi_{1}$ and $\chi_{2}$ two diffeomorphisms of $[0,1]$ onto itself so that $\chi_{j}(x)<x$ on $(0,1), \chi_{j}(x)=$ $x-(x-1)^{2}$ for $x$ close to $1, \chi_{1} \equiv \chi$ and $\chi_{2} \equiv x-x^{p}+\alpha x^{2 p-1}$ for $x$ close to 0 .

We show that $\chi_{1}$ and $\chi_{2}$ are conjugate to each other (say in a neighborhood of 0 in $[0,1])$ by just applying the basic scheme:

Claim. The sequence $\chi_{1}^{(-n)} \circ \chi_{2}^{(n)}$ is convergent in $\mathscr{C}^{\infty}$ topology on $[0, A)$, for every $A<1$.

It is easy to show that (notation being self-explanatory):

(i) For all $x \in[0, A), 0 \leq \chi_{2}^{(n)}(x) \leq \frac{M}{n^{1 /(p-1)}}$ (for some $M>0$ ). (This is detailed in the proof of Lemma 2.)

(ii) $\left\|u \circ v-u_{1} \circ v\right\|_{\mathscr{C} k} \leq c\left\|u-u_{1}\right\|_{\mathscr{C} k}\left(1+\|v\|_{\mathscr{C} k}\right)^{k}$.

And we can take the norm of $u-u_{1}$ on the range of $v$ only.

(iii) $\left\|u \circ v-u \circ v_{1}\right\|_{\mathscr{C}^{k}} \leq C\|u\|_{\mathscr{E} k+1}\left\|v-v_{1}\right\|_{\mathscr{C}_{k}}\left(1+\|v\|_{\mathscr{C}^{k}}+\left\|v_{1}\right\|_{\mathscr{C}^{k}}\right)^{k}$. 
(iv) $\left\|\chi_{1}^{-1} \circ \chi_{2}-\mathbf{1}\right\|_{\mathscr{E} k(0, \varepsilon)}=\mathscr{O}\left(\varepsilon^{\mathbf{K}}\right)$ as $\varepsilon \rightarrow 0$, and $\mathbf{K}$ is arbitrary, and $k$ is fixed.

To establish the claim, write

$$
\chi_{1}^{(-n-1)} \circ \chi_{2}^{(n+1)}-\chi_{1}^{(-n)} \circ \chi_{2}^{(n)}=\chi_{1}^{(-n)} \circ\left(\chi_{1}^{-1} \circ \chi_{2}\right) \circ \chi_{2}^{(n)}-\chi_{2}^{(-n)} \circ \mathbf{1} \circ \chi_{2}^{(n)} .
$$

(i), (ii), (iv), and Lemma 2 show that

$$
\left\|\left(\chi_{1}^{-1} \circ \chi_{2}\right) \circ \chi_{2}^{(n)}-1 \circ \chi_{2}^{(n)}\right\|_{\mathscr{E} k([0, A])}=\mathscr{O}\left(\frac{1}{n^{\mathbf{K}}}\right),
$$

for $\mathbf{K}$ arbitrary. Then (iii) and Lemma 2 show that

$$
\left\|\chi_{1}^{(-n-1)} \circ \chi_{2}^{(n+1)}-\chi_{1}^{(-n)} \circ \chi_{2}^{(n)}\right\|_{\mathscr{C}^{k}([0, A])}=\mathscr{O}\left(\frac{1}{n^{\mathbf{K}}}\right)
$$

(K "different but still arbitrary"). The sequence is therefore converging in all $\mathscr{C}^{k}$ spaces. And obviously its limit $\psi$ coincides with $x$ to infinite order at 0 , and conjugates $\chi_{1}$ to $\chi_{2}$, on some neighborhood of 0 in $\mathbb{R}^{+}$.

One does similarly on the negative axis. However if the point 0 is repulsive for $x-x^{p}$, i.e. if $p$ is even, we should replace $\chi$ and $x-x^{p}+\alpha x^{2 p-1}$ by their inverse (or permute the factors for defining $\psi$ ). At any rate one gets a local change of variable $\psi$, which also coincides with $x$ to infinite order at 0 , which conjugates our two functions near 0 on the negative real axis. The two changes of variable do patch in a $\mathscr{C}^{\infty}$ way and this gives the theorem.

Note. It is certainly not claimed that $\chi_{1}^{(-n)} \circ \chi_{2}^{(n)}$ converges in $\mathscr{C}^{\infty}$ topology on $[0,1]$. See below.

2. Takens has also a theorem on flows (Theorem 1 in [23]).

From the theorem above, it follows that every map $\chi$ as in the statement is locally the time 1 map of the flow of some smooth $\mathscr{C}^{\infty}$ vector field with 0 as rest point. (Therefore $\chi$ has a smooth square root.) Indeed it is sufficient to match $\chi$ to order $2 p-1$ by a time 1 map.

It is interesting to notice that this (germ of) vector field, and not only its Taylor expansion, is unique. This is the simple and clever Proposition 5.1 in [23] (if $\chi=T_{1}^{X}=T_{1}^{X^{\prime}}$, apply Proposition 5.1 to $\varphi=T_{t}^{X} \circ T_{-t}^{X^{\prime}}$ ). See below. So the reduction of functions gives the reduction of vector fields.

3. Remark. There are "many" continuous functions defined near 0 on $\mathbb{R}$ so that $\varphi \circ \varphi=x+x^{2}$. (Given $0<\varepsilon<\varepsilon_{1}<\varepsilon+\varepsilon^{2}$ one can prescribe the restriction of $\varphi$ to $\left[\varepsilon, \varepsilon_{1}\right]$ to be any continuous increasing functions mapping $\varepsilon$ to $\varepsilon_{1}$ and $\varepsilon_{1}$ to $\varepsilon+\varepsilon^{2}$.) The same can be done on the negative axis.

By Takens's argument, mentioned above, there is only one smooth square root. The argument shows in fact uniqueness for roots of class $\mathscr{C}^{2}$. Here we follow the proof of Proposition 5.1 in [23]. Let $\varphi_{1}$ and $\varphi_{2}$ be two (local) square roots of $x+x^{2}$. If $\varphi_{1}$ and $\varphi_{2}$ are of class $\mathscr{C}^{2}, x-\varphi_{1} \circ \varphi_{2}^{-1}(x)=o\left(x^{2}\right)$, by the formal theory.

Let $T_{t}$ denote the smooth (local) flow such that $x+x^{2}=T_{1}$. This is the flow of a smooth vector field $X=x^{2}+\mathscr{O}\left(x^{3}\right)$. For $x$ close to 0 in $\mathbb{R}$, $x \neq 0$, following Takens, define $t(x)$ by $T_{t(x)}(x)=\varphi_{1} \circ \varphi_{2}^{-1}(x)$. One sees that $t(x)=o(1)$, as $x$ tends to 0 . Moreover since $\varphi_{1}$ and $\varphi_{2}$, and therefore $\varphi_{1} \circ \varphi_{2}^{-1}$, commute with $x+x^{2}=T_{1}, t(x)=t\left(x+x^{2}\right)$. Fix $x$, its forwards 
or backwards iterates, under the map $x \mapsto x+x^{2}, x_{n}$ converge to 0 . So $t(x)=t\left(x_{n}\right) \rightarrow 0$. So $t=0$, and $\varphi_{1}=\varphi_{2}$.

4. Proof of Lemma 2. As said earlier we can assume that $\chi(x)<x$ on $(0,1)$, and let $n$ tend to $+\infty$. There exist $p \in \mathbb{N}, p \geq 2$, and $\lambda>0$ so that, at 0 , $\chi(x)=x-\lambda x^{p}+\mathscr{O}\left(x^{p+1}\right)$. Let $\eta \in\left[0, \frac{1}{\lambda p}\right)$ so that, on $[0, \eta], \chi(x) \leq x-\frac{\lambda}{2} x^{p}$; the upper bound $\frac{1}{\lambda p}$ is just to guarantee that $x-\frac{\lambda}{2} x^{p}$ is monotone on $[0, \eta]$. There exists $J$ so that, for every $j \geq J$ and $x \in[0,1 / 2], \chi_{(n)}^{(j)}(x) \in[0, \eta]$; then

$$
\chi^{(j+1)}(x) \leq \chi^{(j)}(x)-\frac{\lambda}{2} \cdot\left[\chi^{(j)}(x)\right]^{p}
$$

One can then easily show by induction on $j$ that there exists $C$ so that for every $x \in[0,1 / 2]$

$$
\chi^{(j)}(x) \leq C j^{-1 /(p-1)} \quad(j \in \mathbb{N}) .
$$

Take $C$ so large that the inequality holds for $0 \leq j \leq J$, and $C \geq\left(\frac{2}{\lambda(p-1)}\right)^{1 /(p-1)}$.

The situation is similar at the point 1 , but we have to use the backwards dynamics (since 1 is repulsive). There exist $q \geq 2$ and $\mu>0$ so that

$$
\chi(x)=x-\mu(1-x)^{q}+\mathscr{O}(1-x)^{q+1} .
$$

And there exists $C_{1}$ so that for every $x \in[1 / 2,1]$

$$
1-\frac{C_{1}}{j^{1 /(q-1)}} \leq \chi^{(-j)}(x) \text {. }
$$

We are now in position of estimating the first derivative of $\chi^{(n)}$.

$$
\left(\chi^{(n)}\right)^{\prime}(x)=\chi^{\prime}\left(\chi^{(j-1)}(x)\right) \cdots \chi^{\prime}\left(\chi^{(j)}(x)\right) \cdots \chi^{\prime}(\chi(x)) \chi^{\prime}(x) .
$$

Let $j_{0}$ be the first integer, such as $\chi^{\left(j_{0}\right)}(x) \leq \frac{1}{2}$; of course $j_{0}$ depends on $x$. For $j \geq j_{0}$ we have

$$
\chi^{(j)}(x) \leq C\left(j-j_{0}\right)^{-1 /(p-1)}
$$

so $\left|1-\chi^{\prime}\left(\chi^{(j)}(x)\right)\right| \leq C^{\prime}\left(j-j_{0}\right)^{-1}$ for some $C^{\prime}$ depending only on $\chi$. For $j<j_{0}, \chi^{(j)}(x)=\chi^{\left(j+1-j_{0}\right)} \chi^{\left(j_{0}-1\right)}(x)$ and $\chi^{\left(j_{0}-1\right)}(x)>\frac{1}{2}$. So

$$
\chi^{(j)}(x) \geq 1-C_{1}\left(j_{0}-j-1\right)^{-1 /(q-1)},
$$

and therefore $\left|1-\chi^{\prime}\left(\chi^{(j)}(x)\right)\right| \leq C^{\prime \prime}\left(j_{0}-j\right)^{-1}$. Using these estimates in the product $(*)$ yields

$$
\left|\left(\chi^{(n)}\right)^{\prime}\right| \leq\left[\prod_{l=1}^{n}\left(1+\max \left(C^{\prime}, C^{\prime \prime}\right) \frac{1}{l}\right)\right]^{2} .
$$

So $\left|\left(\chi^{(n)}\right)^{\prime}\right|=\mathscr{O}\left(n^{s_{1}}\right)$ for some $s_{1}>0$. Now we look at the second derivative $\left(\chi^{(n)}\right)^{\prime \prime}$. It is given by the sum of $n$ terms such as (for $0 \leq j \leq n-1$ )

$$
\chi^{\prime}\left(\chi^{(n-1)}(x)\right) \cdots \chi^{\prime}\left(\chi^{(n-j+1)}(x)\right)\left[\chi^{\prime \prime}\left(\chi^{(n-j)}(x)\right)\left(\chi^{(n-j)}\right)^{\prime}(x)\right] \chi^{\prime}\left(\chi^{(n-j-1)}(x)\right) \cdots .
$$

It is the same product as above with only one factor changed, and $\left(\chi^{(n-j)}\right)^{\prime}$ has already been estimated. And one gets $\left|\left(\chi^{(n)}\right)^{\prime \prime}\right|=\mathscr{O}\left(n^{2 s_{1}+1}\right)$. Using similarly the result just obtained one can estimate $\left(\chi^{(n)}\right)^{\prime \prime \prime}$, etc. 
5. Diffeomorphisms of the closed interval (following J. Robbin). Takens's results are in strong contrast with the results explained further for holomorphic maps. However in the $\mathscr{C}^{\infty}$ category there is a result by J. Robbin, based on N. Kopell's work [11], which offers a striking similitude with the Ecalle-Voronin theory. (Compare the conjugacies on $[0,1)$ and $(0,1]$ with sector conjugacy, and in the Ecalle-Voronin theory 0 serves both as an attractive and a repulsive point.) It comes naturally in the discussion at this point.

We go back to the linearizable case. To make things precise consider a smooth function on $[0,1]$ such that $f(x)<x$ if $x \in(0,1), f(0)=0, f(1)=1$, $f^{\prime}>0, f^{\prime}(0)<1, f^{\prime}(1)>1$.

Following Sternberg one can linearize $f$ at 0 . The linearization $\psi$ extends in fact to a smooth map on $[0,1)$ so that $\psi \circ f(x)=a \psi(x)\left(a=f^{\prime}(0)\right)$. Indeed once we know the convergence of $\frac{f^{n}}{a^{n}}$ on some neighborhood of 0 , the convergence is immediate on the whole basin of attraction. It extends obviously to a diffeomorphism, by using the relation $\psi \circ f=a \psi$. So there is a smooth vector field $X$ on $[0,1)$ so that its time 1 map is $f$. And $X$ is unique (adapt Proposition 5.1 in [23]). Also there is a unique smooth vector field $Y$ on $(0,1]$ whose time $1 \mathrm{map}$ is $f$. The fact that possibly $X \neq Y$ leads to a conjugacy invariant (see $[14$, p. 424]).

The fact that it is possible that $X \neq Y$ is clear. Indeed nonsmooth vector fields may very well have a time 1 map which is smooth. Here we give an explicit example on $\mathbb{R}$ :

$$
X(x)=\frac{x}{1+\frac{\cos (2 \pi \ln |x|)}{2}},
$$

which is smooth except at 0 , and the time 1 map is $T_{1}(x)=e x$.

And here is a way to get this: To solve

$$
\frac{d T_{t}(x)}{d t}=X\left(T_{t}(x)\right), \quad T_{0}(x)=x
$$

one can use separation of variables (not only a computation trick but a proof for conjugacy!; more about this in $§ I V$ )

$$
\frac{d T_{t}}{X\left(T_{t}\right)}=d t, \quad F\left(T_{t}(x)\right)=t+F(x) \quad\left(F^{\prime}=\frac{1}{X}\right) .
$$

The vector field $X(x)=x$ (flow $T_{t}(x)=e^{t} x$ ) corresponds to the equation for the time 1 map $T_{1}(x)=e^{x}$ :

$$
\ln \left|T_{1}(x)\right|=1+\ln |x| .
$$

Now we can add $\rho\left(\ln \left|T_{1}(x)\right|\right)$ to the left-hand side and $\rho(\ln |x|)$ to the right hand side without changing the map $x \rightarrow T_{1}(x)$ implicitly defined provided that $\rho$ has period 1. We choose $\rho(x)=\frac{\sin 2 \pi x}{4 \pi}$. This gives $F(x)=\ln |x|+\frac{\sin 2 \pi(\ln |x|)}{4 \pi}$. Then $X=\frac{1}{F^{\prime}}=\frac{x}{1+\frac{\cos (2 \pi \ln (x))}{2}}$.

6. Takens's theory applies to germs of maps $f$ which are tangent to the identity, at 0 , but such that $f(x)-x$ is not flat at 0 . The reader should look at the paper by F. Sergeraert [17] for the flat case, and at the references mentioned in [17]. 


\section{FIRST, EASY, EXAMPLES}

1. Proposition 4. Let $f(z)$ be any entire function on $\mathbb{C}$ such that $f(z)=$ $z+\sum_{j=2}^{\infty} a_{j} z^{j}$. Then there is no local holomorphic change of variable $\varphi$ defined near $0, \varphi(0)=0$, so that $\varphi^{-1} \circ f \circ \varphi(z)=\frac{z}{1-t z} \quad(t \neq 0$, fixed $)$.

Notice that, as seen in $\S I I, a_{3} / a_{2}^{2}=1$ is a necessary and sufficient condition for formal conjugacy. It is enough to treat the case $t=1$, since the maps $\frac{z}{1-t z}$ and $\frac{z}{1-z}$ are conjugate via the change of variable $z \mapsto t z$.

Proof. Assume that $\varphi$ is defined for $|z|<r$. For every integer $n$ there exists $r_{n}>0$ so that for $|z|<r_{n}, \varphi^{-1} \circ f^{(n)} \circ \varphi=\left(\frac{z}{1-z}\right)^{(n)}=\frac{z}{1-n z}$. So, for $|z|<r_{n}$,

$$
f^{(n)} \circ \varphi(z)=\varphi\left(\frac{z}{1-n z}\right) \text {. }
$$

This is used to extend $\varphi$ on the range of $|z|<r$ under the map $z \mapsto \frac{z}{1-n z}$, since the left-hand side obviously makes sense for $|z|<r$ (and arbitrary $n$ ). More precisely let $\Omega_{0}=\{z \in \mathbb{C},|z|<r\}$. Let $\Omega_{n}$ be the image in the Riemann sphere of $\Omega_{0}$ under the map $z \mapsto \frac{z}{1-n z}$. For $n$ large enough $\Omega_{n}$ is the complement in the Riemann sphere of a small disk $\Delta_{n} \subset \Omega_{0}$.

The inverse of the map $z \mapsto \frac{z}{1-n z}$ is the map $z \mapsto \frac{z}{1+n z}$. On the $\Omega_{n}$ set $\tilde{\varphi}(z)=f^{(n)} \circ \varphi\left(\frac{z}{1+n z}\right)$. Near $0, \tilde{\varphi}=\varphi$, hence everywhere on $\Omega_{0}-\Delta_{n}$ by unique analytic continuation. So $\varphi$ can be extended (by $\tilde{\varphi}$ ) to a holomorphic function on the Riemann sphere. So $\varphi$ is constant, a contradiction.

Corollary. If $f(z)=z+z^{2}+z^{3}+\sum_{j=4}^{\infty} a_{j} z^{j}$ is an entire function, $f(z)$ cannot be in any neighborhood of 0 the time $t$ map of a holomorphic vector field $X=$ $\sum_{j=1}^{\infty} b_{j} z^{j}$ (defined near 0$)$.

Proof. This vector field should be $X=z^{2}+\sum_{j \geq 4} b_{j} z^{j}$. A local change of variable would change it into $z^{2}$ and that would give a conjugacy between $f$ and $\frac{z}{1-z}$, as ruled out by Proposition 3 .

2. Remarks on Voronin's theory. Voronin's theory seems to provide a way to establish Proposition 1 for entire functions along the lines of the proof of Proposition 4. One does not need to take Voronin's theory from the beginning. One can start with Theorem 6.1 in [24].

Let $X=\sum_{j=1}^{\infty} b_{j} z^{j}$. To solve $\frac{d T_{t}(z)}{d t}=X\left(T_{t}(z)\right)$ one uses separation of variables (thus conjugating the flow to a translation), and this leads to

$$
F\left(T_{t}(x)\right)=t+F(x) \quad \text { with } F^{\prime}(z)=\frac{1}{X(z)} .
$$

So $F$ has a singularity at 0 and may not be single valued. The above relation can be written

$$
\frac{1}{F} \circ T_{t}=\frac{z}{1+t z} \circ \frac{1}{F} .
$$

Taking $G=\left(\frac{1}{F}\right)^{-1}$, where possible, one ends with an equivalence relation $f \circ G=G \circ \frac{z}{1-z}$ holding near 0 in some sector. Using again $f^{n} \circ G=G \circ \frac{z}{1-n z}$ one extends $G$ to the complement of 0 in the Riemann sphere. Possibly an additional argument allows one to end the proof. But we feel that details are 
already cumbersome enough so that we prefer to keep the proof of Proposition 1 that we are going to explain. Instead of being based on the dynamics of the vector field $z^{2}$ (i.e. the maps $\frac{z}{1-t z}$ ), it is based on the dynamics of the vector field $z^{p}+C z^{2 p-1}$, whose flow contains a map formally conjugate to the map under study.

\section{BAKER'S AND SZEKERES'S THEOREMS ON FLOWS}

In this section we prove the holomorphic versions of Proposition 1 (Propositions $1.0,1.1)$. As already said in the Introduction these results were first proved by G. Szekeres (entire or rational functions) and I. N. Baker (meromorphic functions), with a different approach. The essential part of our argument is given in Subsection 2. The reader may skip Subsection 3, at the cost of not getting Proposition 1 in full generality.

1. Flow of the vector field $z^{2}+z^{3}$. For simplicity let us first look at the real line in $\mathbb{C}$. Solving

$$
\left\{\begin{array}{l}
\dot{x}=x^{2}+x^{3} \\
x(0)=x_{0} \in \mathbb{R}
\end{array} \quad\left(x=x(t), \dot{x}=\frac{d x}{d t}\right),\right.
$$

leads to

$$
-\frac{1}{x}-\operatorname{Ln}|x|+\operatorname{Ln}|x+1|=t-\frac{1}{x_{0}}-\operatorname{Ln}\left|x_{0}\right|+\operatorname{Ln}\left|x_{0}+1\right| .
$$

The points 0 and -1 are stationary. The points $x_{0}$ in $(0,+\infty)$ and $(-\infty,-1)$ reach respectively $+\infty$ and $-\infty$ in finite time $t=\frac{1}{x_{0}}-\operatorname{Ln}\left|1+\frac{1}{x_{0}}\right|$. The points in $(-1,0)$ flow to 0 (as $t \mapsto+\infty)$.

To understand the situation at $\infty$ (in $\mathbb{C}$ ), set $\zeta=\frac{1}{z}$. The equation $\dot{z}=$ $z^{2}+z^{3}$ transforms into $\dot{\zeta}=-1-\frac{1}{\zeta}$, which has a singularity at $\zeta=0$. By appropriate change of time along each orbit, this vector field has the same orbit as the vector field $|\zeta|^{2}\left(-1-\frac{1}{\zeta}\right)=-\bar{\zeta}-\zeta \bar{\zeta}$, which has 0 as a hyperbolic rest point and the dynamics can therefore be represented in the $\zeta$ coordinate by Figure 1.

Notice however that from the real axis one reaches 0 in finite time (with infinite speed), and in some sense the point then splits into two points which then flow on the outcoming orbits (unstable manifolds!).

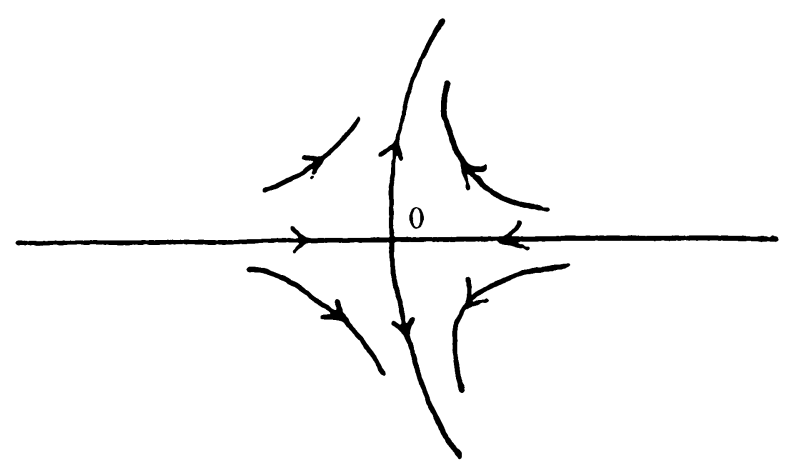

FigURE 1 
In fact it is better to use the results from $\S I I$. A local change of variable at 0 can change the vector field $-\frac{1}{\zeta}-1$ into the vector field $-\frac{1}{\zeta}$. And this amounts to saying that a local change of variable at $\infty$ transforms the vector field $z^{2}+z^{3}$ into the vector field $z^{3}$ whose flow is $T_{t}(z)=\frac{z}{\sqrt{1-t z^{2}}}$, with a cut to be made along $\left(-\infty,-\frac{1}{\sqrt{t}}\right)$ and $\left(\frac{1}{\sqrt{t}},+\infty\right)$ for $t>0$. For $|x|>\frac{1}{\sqrt{t}}$ we have two determinations of $\sqrt{1-t x^{2}}$, leading to two points on the imaginary axis (the two orbits from $\infty$ to 0 for the vector field $z^{3}$ ).

Going through the computations explained in $\S I I$ to reduce the vector field $z^{2}+z^{3}$ to $z^{3}$ at $\infty$ gives us that the orbits flowing out of $\infty$ for the vector field $z^{2}+z^{3}$ have the line $x=-\frac{1}{3}$ as an asymptote. This can be "explained" in another way: Set $z=x+i y, \operatorname{Re}\left(z^{2}+z^{3}\right)=x^{2}-y^{2}+x^{3}-3 x y^{2}$. For $x=-\frac{1}{3}$ the $y^{2}$ terms cancel out and $\operatorname{Re}\left(z^{2}+z^{3}\right)=\frac{2}{27} \cdots$.

Near 0 the vector field $z^{2}+z^{3}$ cannot be reduced to $z^{2}$, as seen in §II. But the action stays "similar" to the action of $z^{2}$ whose orbits are circles, tangent to the real axis at 0 .

Let us sketch the orbits of $z^{2}+z^{3}$. (See Figure 2.)

To analyze the situation several observations may help. The set of points $z$ so that $\operatorname{Re}\left(z^{2}+z^{3}\right) \bar{z}<0$ (this corresponds to $\frac{d}{d t}|z(t)|^{2}<0$ ) is given by the condition $x+x^{2}-y^{2}<0 \quad(z=x+i y)$. Notice that along the hyperbola $x+x^{2}-y^{2}=0$ the vector field points towards the region $x+x^{2}-y^{2}<0$ (the

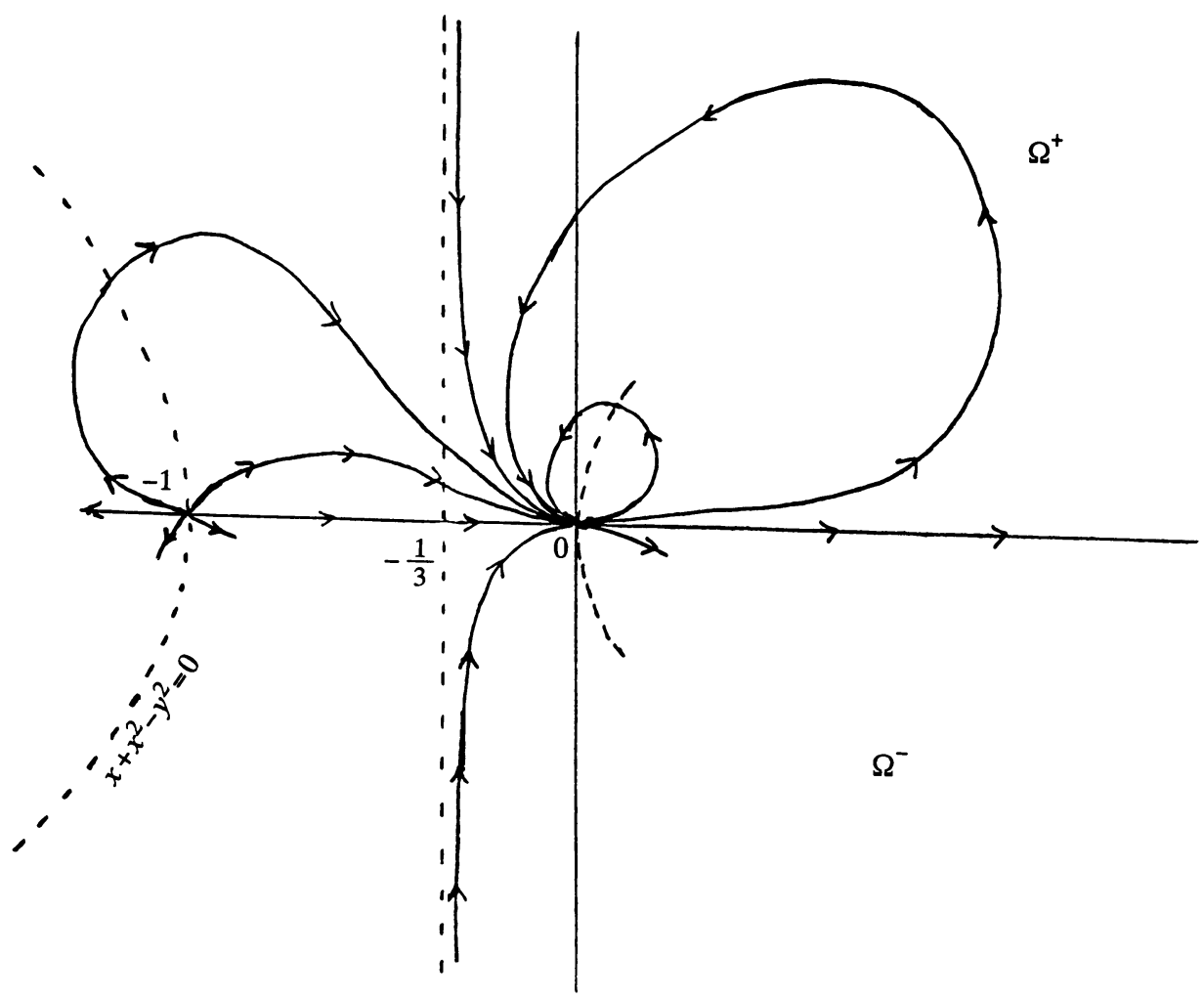

FIGURE 2 
verification is easy taking into account that along the hyperbola the vector field is orthogonal to the radius, and 0 only at the points 0 and -1 ). So a point starting in this region $x+x^{2}-y^{2}<0$ will flow to 0 and the distance to 0 will always decrease (as $t$ increases from 0 to $+\infty$ ), while for a point starting from the region $x+x^{2}-y^{2}>0$, not on the real axis, the distance will first increase, then decrease to 0 after entering the region $x+x^{2}-y^{2}<0$. And remember that at $\infty$ the picture is entirely understood (exact reduction to $z^{3}$ ). Along the line $x=\frac{1}{3}, \operatorname{Re}\left(z^{2}+z^{3}\right)=\frac{2}{27}>0$, and along the $y$-axis $\operatorname{Re}\left(z^{2}+z^{3}\right)<0$ (except at 0 ). This forces the two orbits flowing from $\infty$, in the upper and resp. lower half-plane, to stay in the region $-\frac{1}{3} \leq x \leq 0$. And they flow to 0 , staying in the upper, resp. lower, half-planes.

Other easy observations (such as if $\operatorname{Re} z(t)$ and $\operatorname{Im} z(t)>0, \frac{d}{d t} \arg z(t)>0$ ) can be made.

Here we try to summarize what we will need:

Lemma 3. For every $z_{0}=x_{0}+i y_{0} \in \mathbb{C}, y_{0} \neq 0$ the equation $\dot{z}(t)=z^{2}+z^{3}$, $z(0)=z_{0}$ can be solved for $0 \leq t<+\infty$. We set $T_{t}\left(z_{0}\right)=z(t)$. The map $\left(z_{0}, t\right) \mapsto T_{t}\left(z_{0}\right)$ is continuous in $\left(z_{0}, t\right)$ and holomorphic in $z_{0}$. For fixed $z_{0}$ the function $t \mapsto\left|T_{t}\left(z_{0}\right)\right|$ is either always decreasing to 0 , or increasing and then decreasing to 0 .

If $x_{0}>0$ and $t \neq t_{0}=\frac{1}{x_{0}}-\operatorname{Ln}\left(1+\frac{1}{x_{0}}\right)$ the limits as $(x, y) \mapsto\left(x_{0}, 0^{+}\right)$ (resp. $\left.(x, y) \rightarrow\left(x_{0}, 0^{-}\right)\right)$of $T_{t}(x+i y)$ exist and are denoted by $T_{t}^{+}\left(x_{0}\right)$ (resp. $\left.T_{t}^{-}\left(x_{0}\right)\right)$. For $t \in\left[0, t_{0}\right), T_{t}^{+}\left(x_{0}\right)=T_{t}^{-}\left(x_{0}\right)>0$, but for $t>t_{0}, T_{t}^{+}\left(x_{0}\right) \xi^{+}$ $T_{t}^{-}\left(x_{0}\right)$ and as $t \rightarrow+\infty, T_{t}^{ \pm}\left(x_{0}\right)$ tends to 0 .

$T_{t}^{+}\left(x_{0}\right)$ is a point on the orbit arriving from $\infty$ in the upper half-plane, and $T_{t}^{-}\left(x_{0}\right)=\overline{T_{t}^{+}\left(x_{0}\right)}$.

After the discussions which preceded, we hope that the reader will be easily convinced of the validity of the lemma.

2. Now we are in the position of showing the following.

Lemma 4. Let $C \in \mathbb{R}$. No entire function $f(z)=z+\sum_{j=2}^{\infty} a_{j} z^{j}(f(z) \not \equiv z)$ can be conjugate, under a local holomorphic change of variable at 0 , to the time $t$ map of the vector field $z^{2}+C z^{3}$, for any time $t \in \mathbb{Z}$.

By using the change of variable $z \rightarrow C z$, and changing the time $\left(t \mapsto C t, C \in \mathbb{R}^{*}\right)$ we can assume that $X=z^{2}$, case already treated, or that $X=z^{2}+z^{3}$, case that we will now consider.

Proof. So we are dealing with the vector field $z^{2}+z^{3}$, and the notations are the ones defined in 1.

Let $\Omega^{+}$(resp. $\Omega^{-}$) be the region in the upper (resp. lower) half-plane to the right of the orbits from $\infty$ to 0 .

(i) First we will consider the case $t>0$. Suppose that for $|z|<r<1$, $f \circ \varphi(z)=\varphi \circ T_{t}(z)$ for some local change of variable $\varphi$. For $n>0$ on a "small" neighborhood of $0, f^{(n)} \circ \varphi=\varphi \circ T_{n t}(z)$. But the left-hand side makes sense for $|z|<r$, for all $n \in \mathbb{N}$. Set $\Omega_{r}^{ \pm}=\Omega^{ \pm} \cap\{|z|<r\}$. At least if $r$ is small enough it is clear that $\Omega_{r}^{ \pm}$is connected.

For $z \in T_{n t}\left(\Omega_{r}^{+} \cup \Omega_{r}^{-}\right)$set $\tilde{\varphi}(z)=f^{(n)} \circ \varphi\left(T_{-n t}(z)\right.$ ) (which might a priori depend on $n)$. For $n$ large enough $T_{n t}\left(\Omega_{r}^{ \pm}\right) \cup \Omega_{r}^{ \pm}=\Omega^{ \pm}$. Since $\Omega^{ \pm}$is simply 
connected the intersection $T_{n t}\left(\Omega_{r}^{ \pm}\right) \cap \Omega_{r}^{ \pm}$is connected (however this could be achieved in a more constructive way). Since $\tilde{\varphi}$ and $\varphi$ coincide near $0, \tilde{\varphi}$ and $\varphi$ coincide on the intersection of their domains of definition, by unique analytic continuation. But notice that this has been a global argument, not an argument along a fixed orbit (to avoid monodromy problems).

Now, we can conclude. For $y \in\left(0, \frac{r}{2}\right)$ we have for large $n$

$$
\varphi\left(T_{n t}\left(\frac{r}{2}+i y\right)\right)=f^{(n)}\left(\varphi\left(\frac{r}{2}+i y\right)\right) .
$$

Letting $y$ tend to 0 one gets

$$
\varphi\left(T_{n t}^{+}\left(\frac{r}{2}\right)\right)=f^{(n)} \circ \varphi\left(\frac{r}{2}\right) .
$$

In the same way $\varphi\left(T_{n t}^{-}\left(\frac{r}{2}\right)\right)=f^{(n)} \circ \varphi\left(\frac{r}{2}\right)$. So $\varphi\left(T_{n t}^{+}\left(\frac{r}{2}\right)\right)=\varphi\left(T_{n t}^{-}\left(\frac{r}{2}\right)\right)$, which contradicts the fact that $\varphi$ is one-to-one.

(ii) Case $t<0$ (backwards dynamics). Again define $\tilde{\varphi}$ on $T_{n t}\left(\Omega_{r}^{+} \cup \Omega_{r}^{-}\right)$by

$$
\tilde{\varphi}(z)=f^{(n)} \circ \varphi\left(T_{n|t|}(z)\right) \text {. }
$$

With similar arguments one shows that $\tilde{\varphi}$ and $\varphi$ coincide. For $x \in\left(\frac{r}{4}, \frac{r}{2}\right)$, for $n$ large enough we then get as above

$$
f^{(n)} \circ \varphi\left(T_{n|t|}^{+}(x)\right)=f^{(n)} \circ \varphi\left(T_{n|t|}^{-}(x)\right)=\varphi(x) .
$$

By real analyticity, $n$ being large enough, for any $s>n|t|$ we have

$$
f^{(n)} \circ \varphi\left(T_{s}^{+}\left(\frac{r}{4}\right)\right)=f^{(n)} \circ \varphi\left(T_{s}^{-}\left(\frac{r}{4}\right)\right) .
$$

Indeed, for $s$ close to $n|t|$ write $T_{s}^{ \pm}\left(\frac{r}{4}\right)=T_{n|t|}\left(T_{s-n|t|}\left(\frac{r}{4}\right)\right)$ so the previous equality holds. But $f^{n} \circ \varphi$ is one-to-one on some neighborhood of 0 (which depends on $n)$. Let $s \rightarrow+\infty, T_{s}^{ \pm}\left(\frac{r}{4}\right) \rightarrow 0$, but $T_{s}^{+}\left(\frac{r}{4}\right) \neq T_{s}^{-}\left(\frac{r}{4}\right)$. So a contradiction is reached.

3. We want to generalize Lemma 4 to the case $C \in \mathbb{C}$ (no longer $C$ real).

Lemma 5. Let $C \in \mathbb{C}$. No entire function $f(z)=z+\sum_{j=2}^{\infty} a_{j} z^{j}(\not \equiv z)$ can be conjugate, under a local holomorphic change of variable at 0 , to the time $t$ map of the vector field $z^{2}+C z^{3}$, for any time $t \in \mathbb{R}$.

Instead of giving a detailed proof of Lemma 5 , we will indicate how one can study the dynamics of the vector field $z^{2}+C z^{3} \quad(C \neq 0)$, so that the proof of Lemma 4 is easy to adapt.

Study of the orbits of the vector field $z^{2}+C z^{3} \quad(C \neq 0)$. As previously the situation is entirely clear at $\infty$, since an appropriate change of variable transforms the field in the $z^{3}$ field.

Step 1 (orbits from 0 to $\infty$, and from $\infty$ to 0 , "quadrant" $Q^{+}$).

Claim. There is always an orbit originating at 0 (at time $-\infty$ ) and flowing to $\infty$ (reached in finite time, from every point of the orbit, 0 not included).

Proof of the claim. Let $\omega$ be an arbitrary bounded open neighborhood of 0 . There must be a point $z_{0} \in \omega$ whose forward orbit under the flow reaches $\infty$. If this were not the case, the map $T$ would extend to a map from $\tilde{\omega}$, the forward image of $\omega$ under the flow $\left(\tilde{\omega}=\bigcup_{t \geq 0} T_{t} \omega\right)$, into itself, with 0 as a 
fixed point. The complement of $\tilde{\omega}$ in $\mathbb{C}$ would contain the orbits going to $\infty$. But $T_{1}^{\prime}(0)=1$ and $T_{1} \not \equiv z$. All this is ruled out by Cartan's theorem ([5], or Theorem 3.3 in [10]). Next, near 0 the vector field $z^{2}+C z^{3}$ is "approximated" by $z^{2}$, and one can find a neighborhood $\omega$ of 0 so that every orbit flowing out of $\omega$ has to originate (at time $-\infty$ ) at 0 . This establishes the claim.

The same reasoning applied to the backwards flow shows that: there is also an orbit originating at $\infty$ and terminating (at time $+\infty$ ) at 0 .

These two orbits from $\infty$ to 0 and from 0 to $\infty$ delimit a "quadrant" in $\mathbb{C}$ (one should look at this from $\infty$, the orbits flowing to $\infty$ have asymptotic direction given by $\arg z^{2}=\arg C$, and the orbits coming from $\infty$ have orthogonal asymptotic directions).

We denote by $Q^{+}$this (open) quadrant; $T_{t}$ extends to a map from $Q^{+}$onto $Q^{+}$for all $t \in(-\infty,+\infty)$. (See (ii) below.)

Let us make two remarks:

(i) $Q^{+}$does not contain the rest point $z=-\frac{1}{C}$. If it did, by Schwarz's lemma, and after identification of $Q^{+}$with the unit disk, and of $-\frac{1}{C}$ with 0 , $T_{t}$ would be a rotation, but it is not since 0 (on the boundary of $Q^{+}$) is fixed. As a consequence $Q^{+}$, which is topologically simple, cannot contain any closed orbit.

(ii) There is $\mathscr{O}$, a neighborhood of $\infty$ so that every orbit going through $\mathscr{O} \cap Q^{+}$has to flow to 0 : by continuous dependence on initial data and the fact that every orbit entering the region $\{|z|<\rho\}$ has to terminate at 0 , if $\rho$ is small enough. (Remember: at $\infty$ the situation is completely known.)

Now consider an arbitrary orbit in $Q^{+}$. As just seen it cannot approach $\infty$, so it stays in a compact set in $\mathbb{C}$. By the Poincaré-Bendixson theorem [8] its limit set can be a rest point or a closed orbit. The only possibility left is that the orbit flows to 0 (reached only at time $+\infty$ ).

We hope that the reader is now convinced that $Q^{+}$provides us with a substitute for $\Omega^{+}$used in the proof of Lemma 4.

Step 2. For $R$ large enough the circle $\{|z|=R\}$ can be decomposed in four arcs $\gamma_{1}, \ldots, \gamma_{4}$. Along $\gamma_{1}$ and $\gamma_{3}$ the vector field $z^{2}+C z^{3}$ points towards the region $|z|>R$, along $\gamma_{2}, \gamma_{4}$ it points towards $|z|<R$.

For the vector field $z^{3}$ (to which one can reduce) this corresponds to

$$
\begin{aligned}
& \gamma_{1}=\left\{R e^{i \theta},-\frac{\pi}{4} \leq \theta \leq+\frac{\pi}{4}\right\}, \\
& \gamma_{2}=\left\{R e^{i \theta}, \frac{\pi}{4} \leq 0 \leq \frac{3 \pi}{4}\right\}, \text { etc. }
\end{aligned}
$$

Let $\lambda$ be any closed arc on an orbit of the flow with endpoints $a, b \in \gamma_{j}$ (same $j$ ) with no other intersection with $\gamma_{j}$. Denote by $\omega$ the (simple) region bounded by $\lambda$ and the subarc of $\gamma_{j}$ from $a$ to $b$.

The aim of Step 2 is just to state the following: either for $t>0, T_{t} \omega \nsubseteq \omega$, or for $t<0, T_{t} \omega \nsubseteq \omega$.

Indeed the forward flow can either enter and only enter $\omega$ along $\gamma_{j}$, or leave and only leave.

Step 3. From now on we will assume $\operatorname{Re} C \leq 0$. There is no loss of generality since one can achieve this by reversing time and changing $z$ into $-z$ if needed. Then the linearization of the vector field $z^{2}+C z^{3}$ at $z=-\frac{1}{C}$ (the rest point) 
has nonnegative real part. So no orbit can flow to $-\frac{1}{C}$. Let $\mathscr{S}$ be the remaining orbit coming from $\infty$ (the one not used for defining the boundary of $Q^{+}$).

Claim. The orbit $\mathscr{S}$ has to flow to 0 or to $\infty$.

We first notice that for $R$ large enough the orbit $\mathscr{S}$ cannot cross the circle $|z|=R$ more than four times.

Indeed if it were crossing the circle five times or more, some points of intersection would belong to the same arc $\gamma_{j}\left(\gamma_{j}\right.$ as in Step 2). This would give us a region $\omega$ bounded by a portion of $\mathscr{S}$ and so that $T_{t}(\omega) \nsubseteq \omega$ for $t>0$. Indeed, since $\mathscr{S}$ originates at $\infty$ it is clearly impossible that $T_{t}(\omega) \nsubseteq \omega$ for $t<0$. The region $\omega$ would contain a rest point, i.e. $z_{0}=0$ or $-\frac{1}{C}$. But by Schwarz's lemma we should have $\left|T_{t}^{\prime}\left(z_{0}\right)\right|<1$, for $t>0$, but $T_{t}^{\prime}(0)=1$ and $\left|T_{t}^{\prime}\left(-\frac{1}{c}\right)\right| \geq 1$ (for $\left.t>0\right)$.

We can conclude that either the orbit $\mathscr{S}$ goes to $\infty$ or it stays bounded. Let us assume that it stays bounded.

The orbit $\mathscr{S}$ cannot approach a periodic orbit, because due to holomorphicity the set of periodic (nonrest) points is open (if $T_{t_{0}}\left(z_{0}\right)=z_{0}, T_{t_{0}}(z)=z$ along the orbit, hence in a neighborhood). By the Poincaré-Bendixson theorem the orbit must go to 0 , the only accessible rest point.

Step 4. If the orbit $\mathscr{S}$ flows to 0 , it provides us with a "quadrant" $Q^{-}$(similar to the quadrant $Q^{+}$) which we can substitute to $\Omega^{-}$to prove Lemma 4 along the lines of the proof of Lemma 3.

Step 5. But it can happen that the orbit $Y$ coming from $\infty$ flows back to $\infty$ (in finite time).

The orbit $\mathscr{S}$ isolates then a "quadrant" $Q=$. The flow $T_{t}$ extends to a topological flow on $\overline{Q^{=}} \cup\{\infty\}$ without fixed point on the boundary. So $Q^{=}$ must contain the fixed point $-\frac{1}{C}$, which, by what has been said in Step 1, must be the center of a "rotation". (So $C$ must be pure imaginary, and it is not hard to see that $Q^{=}$is the union of the periodic orbits of the flow.)

The situation is sketched in Figure 3 which corresponds to the vector field $z^{2}+i z^{3}$. In this figure $\Gamma_{1}$ is the orbit from 0 to $\infty, \Gamma_{2}$ is the orbit from $\infty$ to $\infty, \Gamma_{3}$ is the orbit from $\infty$ to 0 . Let $\mathscr{R}^{-}=\mathbb{C}-\bar{Q}^{+} \cup Q^{=}$. Notice that $\mathscr{R}^{-}$does not contain any rest point. By the arguments used in Steps 2 and 3, every orbit in $\mathscr{R}^{-}$must terminate at 0 .

The region $\mathscr{R}^{-}$will be used instead of $\Omega^{-}$in Lemma 4 , but the dynamics are somewhat different. Lemma 3 has to be modified:

For $z_{0} \in \Gamma$, we define $T_{t}^{+}\left(z_{0}\right)$ and $T_{t}^{-}\left(z_{0}\right)$ in the following way:

- For $t \in\left[0, t_{0}\right), T_{t}^{+}\left(z_{0}\right)=T_{t}^{-}\left(z_{0}\right)=T_{t}\left(z_{0}\right) \in \Gamma_{1}$.

- At time $t_{0}, T_{t}^{ \pm}\left(z_{0}\right)$ reaches $\infty$.

- For $t>t_{0}, T_{t}^{+}\left(z_{0}\right)$ flows to 0 along $\Gamma_{3}$ (0 reached only at time $\left.+\infty\right)$.

- For $t_{0}<t<t_{1}, T_{t}^{-}\left(z_{0}\right)$ flows from $\infty$ to $\infty$ (in finite time) along $\Gamma_{2}$.

- Finally $T_{t}^{-}\left(z_{0}\right)$ flows from $\infty$ to 0 as $t$ increases from $t_{1}$ to $+\infty$.

So $T_{t}^{ \pm}\left(z_{0}\right)$ both approach 0 along $\Gamma_{3}$, but $T_{t}^{-}\left(z_{0}\right)$ is delayed $\left(\left(t_{1}-t_{0}\right)\right.$ in time), and $T_{t}^{+}\left(z_{0}\right) \neq T_{t}^{-}\left(z_{0}\right)$ for large $t$.

So we can still reach contradictions as in the proof of Lemma 4, to establish Lemma 5. 


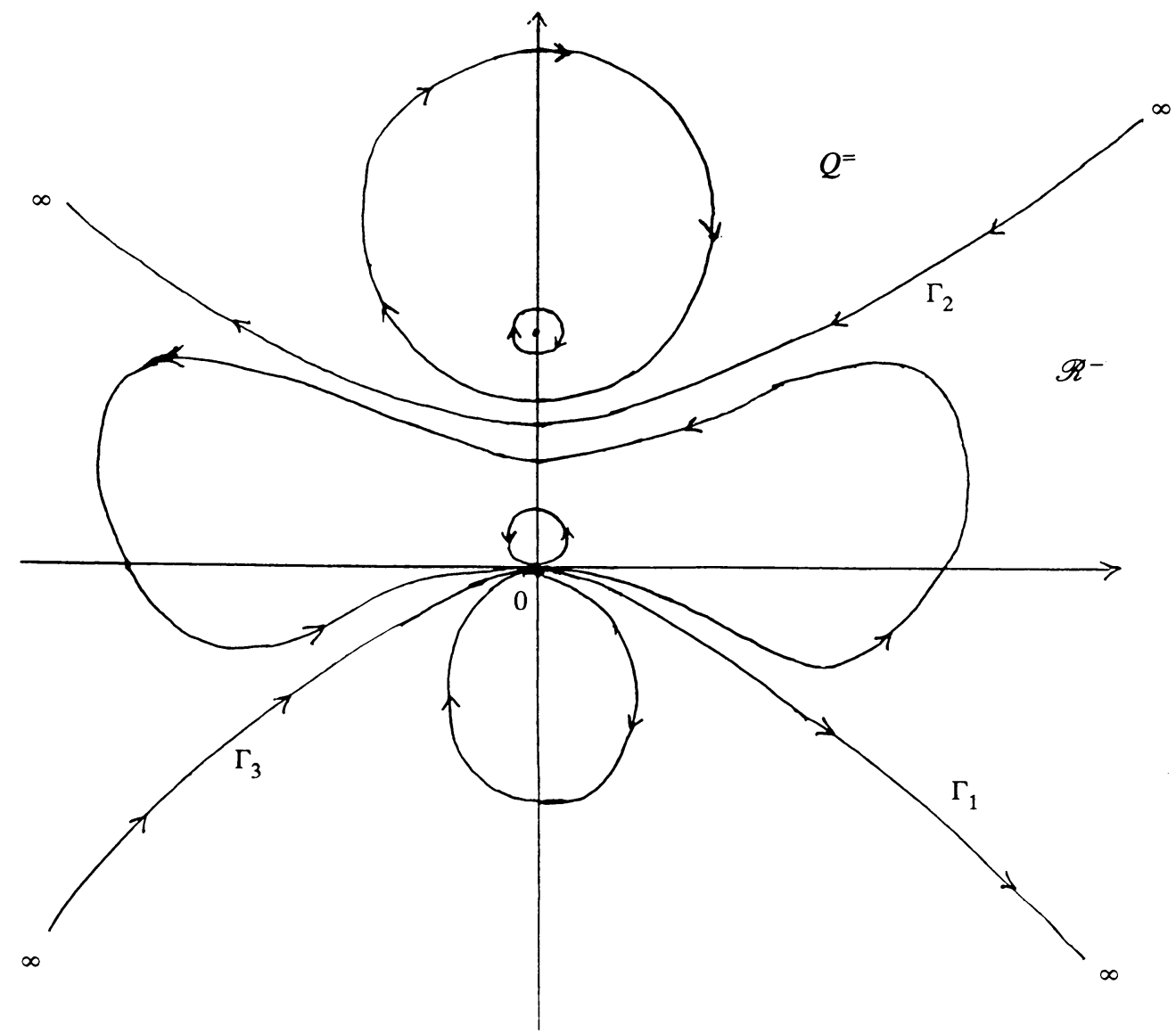

FIGURE 3

Proposition 5. Let $p \geq 2$ and $c \in \mathbb{C}$. Then no entire function

$$
f(z)=z+\sum_{j=2}^{\infty} a_{j} z^{j} \quad(f(z) \not \equiv z)
$$

can be conjugate, under a local holomorphic change of variable at 0 , to the time $t$ map of the vector field $z^{p}+C z^{2 p-1}$, for any time $t \in \mathbb{R}$.

Proof. The case $p=2$ is covered by Lemmas 4 and 5. Although we will not give all the details, there is a simple way to reduce to the case $p=2$. Notice that $z^{p}+C z^{2 p-1}$ is the push forward of the vector field $(p-1)\left(z^{2}+C z^{3}\right)$, under the (multivalued) map $z \mapsto z^{1 /(p-1)}$. So the flow of the vector field $z^{p}+C z^{2 p-1}$ is simply obtained by lifting the flow of $z^{2}+C z^{3}$ to the ramified cover of $\mathbb{C}$ by $z \mapsto z^{p-1}$. So the study of the dynamics does not need to be done again. And Proposition 5 can be obtained along the lines of the proof of Lemmas 4-5. Notice however that the case $C=0$ has to be treated in the same way (using the dynamics of $z^{p}, p \geq 3$ ), not specially as by Proposition 4 . 


\section{Proposition 1.0, entire functions.}

Proposition 1.0. Let $f(z)=z+\sum_{j=p+1}^{\infty} a_{j} z^{j} \not \equiv z$ be an entire function. Then $f$ cannot be a time $t$ map of the flow of a holomorphic vector field

$$
X=\sum_{j \geq 1} b_{j} z^{j}
$$

Proof. If $b_{1}$ were different from 0 , a local change of variable would change $X$ in the vector field $b_{1} z$ so $f$ would be linearized, which is not the case. (The same kind of argument is repeated, more detailed, below.)

So we assume that

$$
X=\sum_{j \geq p} b_{j} z^{j}, \quad p \geq 2, \quad b_{p} \neq 0 .
$$

It follows from $\S$ II that there would exist a local change of variable so that $\varphi_{*}(X)=z^{p}+C z^{2 p-1}$ for some $C \in \mathbb{C}$ so $f$ would be conjugate to the time $t$ map of the flow of the vector field $z^{p}+C z^{2 p-1}$, and this is impossible due to Proposition 5.

Notice that the case of a function

$$
f(z)=z+a_{2} z^{2}+a_{3} z^{3}+\sum_{j=p+1}^{\infty} a_{j} z^{j}
$$

relies only on Lemma 4 if $a_{2} \neq 0$ and $\frac{a_{3}}{a_{2}^{2}} \in \mathbb{R}$. And the case

$$
f(z)=z+\sum_{j \geq p} a_{j} z^{j}, \quad p \geq 2, \quad a_{p} \neq 0,
$$

$a_{p}, a_{p+1}, \ldots, a_{2 p-1}$ real, does not need Lemma 5 .

5. We will now prove a proposition slightly more general than Proposition 1.0, namely:

Proposition 1.1. Let $A$ be a countable closed subset of $\mathbb{C}, 0 \notin A$. Let $f$ be a holomorphic function on $\mathbb{C}-A, f(z)=a z+\sum_{j=2}^{\infty} a_{j} z^{j}$ with $a^{n}=1$ for some positive integer $n$. The following are equivalent:

(i) there exists $X$ a holomorphic vector field $X=\sum_{j=1}^{\infty} b_{j} z^{j}$ defined near 0 , so that near $0 \quad f$ is the time $t$ map of $X$ for some time $t$.

(ii) $f(z)=\frac{a z}{1+b z}$, for some $b \in \mathbb{C}$.

And if $a \neq 1$, (i) and (ii) are also equivalent to

(iii) $f$ is linearizable at 0 .

(iv) $f^{(n)}=f \circ \cdots \circ f \equiv z$.

The class of nonconstant functions holomorphic on the complement of a closed countable set (which may depend on the function) is natural from the point of view of compositions. It contains all nonconstant meromorphic functions and is closed under composition. Moreover we have a Picard theorem for this class: if $f$ is holomorphic in the complement of a closed countable set $A$ and $a \in A$ is not an isolated point of $A$, then either $f$ extends to be meromorphic in a neighborhood of $a$ or $f$ omits at most two values in every neighborhood of $a$. This is an easy consequence of Picard's theorem for 
meromorphic function [16, (VII.13.15)], (or [10, p. 79]). Now we turn to the proof.

Proof. The case $a^{n}=1$ but $a \neq 1$ is easy to treat. From this we see that $b_{1} \neq 0$ and hence it follows that the normal form of $X$ near 0 is $b_{1} z$. This means that $f$ is linearizable; that is, there is a biholomorphic map $\varphi$, defined near 0 , with $\varphi(0)=0$ and $f(\varphi(z))=\varphi(a z)$, from which it follows that $f^{(n)}(\varphi(z))=\varphi(z)$, or $f^{(n)}(z) \equiv z$ near 0 . Now $f$ and $f^{(n)}$ are defined in a set $\mathbb{C} \backslash B$ where $B$ is closed and countable. Clearly $f$ is one-to-one in $\mathbb{C} \backslash B$. By our Picard theorem, any nonisolated points of $B$ are removable, and there can be no isolated essential singularities, and at most one pole, which must have order 1 , including at $\infty$. It follows that $f$ has the required form.

We are still in the case $a \neq 1$. The last part of the argument in the above paragraph shows that (iv) $\Rightarrow$ (ii) and a direct calculation shows that (ii) $\Rightarrow$ (iv). We have just shown that (i) $\Rightarrow$ (ii). We have already noted the equivalence of (i) and (iii) (for any $a \neq 1$ ). It remains to show that $f(z)=\frac{a z}{1+b z}$ is a time 1 map. Clearly $f_{0}(z)=a z$ is a time 1 map; hence if $\varphi(z)=\frac{z}{1+\beta z}, \varphi f_{0} \varphi^{-1}$ is a time 1 map. But $\varphi f_{0} \varphi^{-1}(z)=\frac{a z}{1+b z}$ if $\beta=\frac{b}{1-a}$.

Now we suppose that $a=1$. As we have seen, either $f(z) \equiv z$ (i.e. $b=0$ ) or

$$
X(z)=\sum_{j=2}^{\infty} b_{j} z^{j} .
$$

If the normal form for $X$ near 0 is $z^{p}+\lambda z^{2 p-1}$ with $p>2$, or $z^{2}+C z^{3}$ with $C \neq 0$, we proceed as before to deduce that this is impossible. It must be checked that it is not essential that $f$ be entire; any $f$ satisfying our hypothesis will do. All that remains is an appropriate version of Proposition 4: If $f$ is holomorphic in $\mathbb{C} \backslash A$ for a closed countable $A, f(z)=z+\sum_{j=2}^{\infty} a_{j} z^{j}$ near 0 and $f(\varphi(z))=\varphi\left(\frac{z}{1-z}\right)$ for some $\varphi$, biholomorphic near 0 with $\varphi(0)=0$ then $f(z)=\frac{z}{1+b z}$ for some $b \in \mathbb{C}$.

As before we may iterate the identity $f(\varphi(z))=\varphi\left(\frac{z}{1-z}\right)$, valid near 0 , to obtain an analytic continuation of $\varphi$ into a set of the form $\mathbb{C} \backslash B, B$ countable and closed. Next we argue that $\varphi$ and $f$ must be rational. Indeed, if $\varphi$ were not rational then by Picard's theorem there would be a point $a$ such that $\varphi$ assumes all values, with at most two exceptions, in every neighborhood of $a$. Choose $n$ so that $\frac{a}{1-n a}$ lies in the disc about 0 in which we know $\varphi$ to be holomorphic. We have $f^{(n)}(\varphi(z))=\varphi\left(\frac{z}{1-n z}\right)$. We may choose distinct $z_{k} \rightarrow a$ such that $\varphi\left(z_{k}\right)=\alpha$ where $\alpha$ lies in the domain of $f^{(n)}$. We get $f^{(n)}(\alpha)=\varphi\left(\frac{z_{k}}{1-n z_{k}}\right)$ for all $k$. This would imply $\varphi$ is constant, which is not the case. This argument works for $a=\infty$ as well, so $\varphi$ is rational. Now if $f$ were not rational there would be a sequence of distinct points $\omega_{k}$ in the domain of $f$ such that $f\left(w_{k}\right) \equiv \alpha$. Since $\varphi$ is rational there are $z_{k}$ (distinct) so that $\varphi\left(z_{k}\right)=w_{k}$; we get $\alpha=f\left(\varphi\left(z_{k}\right)\right)=\varphi\left(\frac{z_{k}}{1-z_{k}}\right)$. Again this would imply that $\varphi$ is constant. Now $f, \varphi$ are rational, the identity $f(\varphi(z))=\varphi\left(\frac{z}{1-z}\right)$ implies that either $f$ or $\varphi$ must be one-to-one; otherwise the multiplicity of $f(\varphi(z))$ would be greater than that of $\varphi\left(\frac{z}{1-z}\right)$.If $f$ is one-to-one it must have the desired form. If $\varphi$ is one-to-one, then so is $f$ and we are done.

To complete the equivalence of (i) and (ii) in the case $a=1$ we need only note that $f(z)=\frac{z}{1+b t}$ is the time 1 map of $X(z)=-b z^{2}$. 
VI. THE FUNCTION $z+z^{2}$

1. We will explain in Subsection 3 below that some "monodromy" or "single valuedness" problems make discrete dynamics somewhat harder to use than the continuous dynamics involved in flows. For some specific functions these problems can be overcome. Just as an illustration of this, we present here a study of the function $z+z^{2}$. The following lemma will help to avoid monodromy problems later.

Lemma 6. 1. For every $z \in \mathbb{C}^{*}$, if $\left|z+z^{2}\right| \geq|z|$, then at least one of the two roots $\zeta$ to the equation $\zeta+\zeta^{2}=z$ satisfies $|\zeta|<|z|$.

2. If $R>0,|z| \leq R$, and $\left|z+z^{2}\right| \leq R$, then for every $\lambda \in[0,1], \mid \lambda z+$ $\lambda^{2} z^{2} \mid \leq R$ (i.e. the set of $z$ such that $|z| \leq R$ and $\left|z+z^{2}\right| \leq R$ is star shaped). Proof. 1. If $\left|z+z^{2}\right| \geq|z|$ then $|z+1| \geq 1$. If $|z|>1$ it is trivial that one of the two roots must satisfy $|\zeta|<|z|$ since the product of the roots is $-z$. This leaves us to examine the case $z \neq 0,|z| \leq 1$, and $|z+1| \geq 1$. If $z$ belongs to the region thus defined, one of the two roots belongs to the right half-plane $\operatorname{Re} z \geq 0$ (whose image is the region $\operatorname{Re} z \geq-(\operatorname{Im} z)^{2}$ ). If $\operatorname{Re} \zeta \geq 0, \zeta \neq 0$ then $\left|\zeta+\zeta^{2}\right|>|\zeta|$. So the root $\zeta$ with nonnegative real part satisfies $|\zeta|<|z|$ as desired.

2. Set $z=\rho e^{i \theta}, 0 \leq \rho \leq R$. We have $\left|z+z^{2}\right|^{2}=\rho^{2}+\rho^{4}+2 \rho^{3} \cos \theta$. If the lemma were not true there would exist $r \in[0, R]$ such that

$$
\left\{\begin{array}{l}
r^{2}+r^{4}+2 r^{3} \cos \theta=R^{2}, \\
\frac{1}{2} \frac{d}{d r}\left(r^{2}+r^{4}+2 r^{3} \cos \theta\right)=r+2 r^{3}+3 r^{2} \cos \theta \leq 0 .
\end{array}\right.
$$

(**) implies $r<1 \quad(r=1, \cos \theta=-1$ being ruled out by $(*))$. So assume $r<1$ since $r^{4}+2 r^{3} \cos \theta \geq 0$ by $(*)$ we get

$$
r+2 r^{3}+3 r^{2} \cos \theta=\frac{1}{r}\left(2 r^{4}+4 r^{3} \cos \theta\right)+\left(r-r^{2} \cos \theta\right)>0,
$$

which contradicts $(* *)$. The lemma is proved.

Lemma 7. Let $h$ be a germ of a holomorphic function at 0 in $\mathbb{C}$. Let $G$ be an entire function. If $G \circ h=h \circ F_{0}, F_{0}=z+z^{2}$, then, for some integer $n>0$, $G^{(n)} \circ h$ extends to an entire function on $\mathbb{C}$.

Note that this is only a step in the proof. If $h(0)=0$, and $h^{\prime}(0) \neq 0$, Proposition 2.0 will show that necessarily $G(z)=z+a z^{2}$, for some $a \neq 0$. In case $a=1$, the function $h$ has to be $F_{0}^{(k)}$ for some integer $k$, possibly negative. This follows from Baker's work, or from Theorem 6.3 in [24], once the nonexistence of roots is known. A linear change of variables reduces the general case to the case $a=1$.

Proof. (i) First, we can extend $h$ holomorphically to the right half-plane $\pi^{+}=$ $\{z \in \mathbb{C}, \operatorname{Re} z>0\}$. Indeed it is enough to show that if $h$ has been extended to $\{|z|<R<+\infty\} \cap \pi^{+}$, we can extend $h$ to $\left\{|z|<R^{\prime}\right\} \cap \pi^{+}$for some $R^{\prime}>R$. Let $z_{0}=R e^{i \theta}, \theta \in\left(-\frac{\pi}{2}, \frac{\pi}{2}\right)$. There exists $z_{1} \in\{|z|<R\} \cap \bar{\pi}^{+}$so that $z_{1}+z_{1}^{2}=z_{0}$. For every $\lambda \in(0,1), \operatorname{Re}\left(\lambda z_{1}+\lambda^{2} z_{1}^{2}\right)>0$, so, by Lemma 6, $\lambda z_{1}$ and $\lambda z_{1}+\lambda^{2} z_{1}^{2} \in\{|z|<R\} \cap \pi^{+}$. From this we conclude that the relation $h\left(z+z^{2}\right)=G(h(z))$ holds on some neighborhood of the line segment $\left[0, z_{1}\right)$ since it holds near 0 , by unique analytic continuation. The map $z \mapsto z+z^{2}$ 
defines a biholomorphic map $\tau$ from a neighborhood of $z_{1}$ to a neighborhood of $z_{0}$. We extend $h$ to a neighborhood of $z_{0}$ by setting

$$
h(z)=G\left(h\left(\tau^{-1}(z)\right)\right) .
$$

(ii) In the same way, we can extend $h$ to the set $U^{+}=\left\{z, \operatorname{Re} z>-(\operatorname{Im} z)^{2}\right\}$. Notice that $U^{+}$is the injective image of $\pi^{+}$under the map $z \mapsto z+z^{2}$. Again one defines $h$ at the point $\left(z+z^{2}\right)$ by $h\left(z+z^{2}\right)=G(h(z))$.

(iii) The strategy for $\pi^{-}=\{z \in \mathbb{C}, \operatorname{Re} z<0\}$ will be different. We will not try to extend $h$, but $G^{(m)} \circ h$ instead.

Claim. For every $R>0$ there exists $n \in \mathbb{N}^{*}$ so that $G^{(n)} \circ h$ has a holomorphic extension to $\{|z|<R\}$.

The claim is established by showing that if $G^{(m)} \circ h$ is extended to $\left\{|z|<R_{m}\right\}$, then $G^{(m+1)} \circ h$ extends to $\left\{|z|<R_{m+1}\right\}$, where $R_{m+1}>R_{m}$ and $R_{m+1}-R_{m}$ can be estimated so that to force $R_{m} \rightarrow+\infty$. Here is the way to do it, but we leave details to the reader.

If $\left|z_{0}\right|=R$, due to Lemma 6 at least one of the two following things happens: There exists $z_{1},\left|z_{1}\right|<R$, so that $z_{1}+z_{1}^{2}=z_{0}$ or $\left|z_{0}+z_{0}^{2}\right|<R$ (example $\left.-1<z_{0}<0\right)$. In both cases we use the formula

$$
G^{(m)} \circ h\left(z+z^{2}\right)=G^{(m+1)} \circ h(z) .
$$

In the first case $z_{1} \neq-\frac{1}{2}$, so $z \mapsto z+z^{2}$ is $1-1$ near $z_{1}$, and the above formula is used to extend $G^{(m)} \circ h$ near $z_{0}$ by taking $z$ close to $z_{1} ; G^{(m+1)} \circ h$ is a fortiori extended. In the second case we take $z$ close to $z_{0}$ and we extend only $G^{(m+1)} \circ h$. Notice that by unique analytic continuation, and by Lemma 6 , the equality $G^{(m)} \circ h\left(z+z^{2}\right)=G^{(m+1)} \circ h(z)$ holds on the ray from 0 to $z_{1}$ in the first case, and on the ray from 0 to $z_{0}$ in the second case.

(iv) Finally for some $m$ (we may depend on $\rho$ ) we obtained that $G^{(m)} \circ h$ has a holomorphic extension to $U^{+} \cup\{|z|<1\}$, since the extensions obtained in (ii) and (iii) must coincide. Since $G^{(m+1)} \circ h=G^{(m)} \circ h \circ F_{0}$, say in a neighborhood of $\left(-\frac{1}{2}, 0\right)$, we see that for $\zeta \simeq-\frac{1}{2}, G^{(m+1)} \circ h(\zeta)=G^{(m+1)} \circ h(-1-\zeta)(-1-\zeta$ is the other root to $\left.z+z^{2}=\zeta+\zeta^{2}\right)$. The set of $\zeta$ so that both $\zeta$ and $-1-\zeta$ both belong to $U^{+} \cup\{|z|<1\}$ is connected. So $G^{(m+1)} \circ h(\zeta)=G^{(m+1)} \circ h(-1-\zeta)$ everywhere (where it makes sense). If $\zeta \in \mathbb{C}$ but $\zeta \notin U^{+} \cup\{|z|<1\}$, then $(-1-\zeta) \in U^{+} \cup\{|z|<1\}$. So $G^{(m+1)} \circ h$ can be extended to $\mathbb{C}$ by setting $G^{(m+1)} \circ h(\zeta)=G^{(m+1)} \circ h(-1-\zeta)$.

2. Proposition 2.0. The only entire functions $G$ which are in some neighborhood of 0 holomorphically conjugate to $F_{0}\left(=z+z^{2}\right)$ (i.e. $z+z^{2}=\varphi^{-1} \circ G \circ \varphi$, for some local holomorphic change of variable $\varphi(\varphi(0)=0)$ ) are $f(z)=$ $z+a z^{2}$, for $a \neq 0$.

For $k>1$, in no neighborhood of 0 does the map $z+z^{2}$ have a $k$ th root (i.e. $\left.z+z^{2}=g^{(k)}\right)$, with $g$ holomorphic, and $g(0)=0$.

The nonexistence of roots has also been noticed, earlier, by I. N. Baker.

Proof. Assume that near $0, G \circ \varphi=\varphi \circ F_{0}$ for some local holomorphic change of variable $\varphi$, and that $G$ is entire. By Lemma 7 for some $n$ large enough $G^{(n)} \circ \varphi$ extends to an entire function on $\mathbb{C}$. We have $G \circ\left(G^{(n)} \circ \varphi\right)=\left(G^{(n)} \circ \varphi\right) \circ F_{0}$. 
So this means that if $G$ and $F_{0}$ are conjugate near 0 , via holomorphic change of variable, one can find an entire function $\varphi_{1}, \varphi_{1}(0)=0, \varphi_{1}^{\prime}(0) \neq 0$, so that

$$
G \circ \varphi_{1}=\varphi_{1} \circ F_{0} .
$$

Next, we have

Lemma 8. Let $G$ and $\varphi_{1}$ be nonconstant entire functions. If $G \circ \varphi_{1}=\varphi_{1} \circ\left(z+z^{2}\right)$ then $G$ must be a polynomial of degree 2 .

Proof. To reach a contradiction assume that $G$ is not a polynomial of degree 2. Then $\varphi_{1}$ cannot be a polynomial, by degree considerations. Hence there exists $z_{0} \in \mathbb{C},\left|z_{0}\right|>2$, so that $G \circ \varphi_{1}\left(z_{0}\right)$ belongs to the basin of attraction to $\varphi_{1}(0)$ for $G$ (example: ask $G \circ \varphi_{1}\left(z_{0}\right)=G \circ \varphi_{1}(x)=\varphi_{1}\left(x+x^{2}\right)$ for some $x \in(-1,0))$.

Let $\omega$ be a neighborhood of $z_{0}$ so that $G^{(n)} \circ \varphi_{1}$ tends to $\varphi_{1}(0)$ on $\omega$ as $n$ tends to $\infty$.

We leave to the reader to check that for $n$ large enough the image of $\omega$ under the $n$th iterate of the map $\left(z+z^{2}\right)$ contains a circle $\left\{|z|=R_{n}\right\}$ and $R_{n} \rightarrow+\infty$ (here we use $\left|z_{0}\right|>2$ ). Using $G^{(n)} \circ \varphi_{1}=\varphi_{1} \circ\left(z+z^{2}\right)^{(n)}$, one has

$$
\sup _{|z| \leq R_{n}}\left|\varphi_{1}\right| \leq \sup _{\omega}\left|G^{(n)} \circ \varphi_{1}\right| \rightarrow\left|\varphi_{1}(0)\right| .
$$

So $\varphi_{1}$ would be constant. Lemma 8 is proved, and we have thus showed that the functions $z+a z^{2} \quad(a \neq 0)$ are the only entire functions that are conjugate to $z+z^{2}$ under a local holomorphic change of variable at 0 .

The nonexistence of $k$ th roots is easy to get. In order to reach a contradiction, assume that there exists $g$ defined for $|z|<\rho$, so that in some neighborhood of $0, g^{(k)}(z)=z+z^{2}, k \geq 2$. Obviously $g$ and $z+z^{2}$ must commute. So, by applying Lemma 7 to $G=z+z^{2}=F_{0}$ and $h=g$, one sees that for some integer $n, F_{0}^{(n)} \circ g$ is an entire function.

Since $\left(F_{0}^{(n)} \circ g\right)^{(k)}=F_{0}^{(k n+1)}$ is a polynomial $F_{0}^{(n)} \circ g$ must be a polynomial (all points identified by $F_{0}^{(n)} \circ g$ are identified by $\left.\left(F_{0}^{(n)} \circ g\right)^{(k)}\right)$.

If $F_{0}^{(n)} \circ g$ is a polynomial of degree $r,\left(F_{0}^{(n)} \circ g\right)^{(k)}$ is a polynomial of degree $r^{k}$. But $F_{0}^{(k n+1)}$ is of degree $2 \cdot 2^{k n}$ which is not the $k$ th power of an integer.

3. Comments. If $f$ and $g$ are two germs of holomorphic functions at 0 , $f(0)=g(0)=0, f^{\prime}(0) \neq 0, g^{\prime}(0) \neq 0$, then $f \circ g$ and $g \circ f$ are obviously conjugate to each other (locally).

This explains why, above, we have been careful about monodromy questions. Suppose $f$ and $g$ are entire and $f=z+\cdots, g=z+\cdots$ (both $\not \equiv z)$. If we have a local conjugacy, $f \circ \varphi=\varphi \circ g$. By using $f^{(n)} \circ \varphi=\varphi \circ g^{(n)}$ it seems that one could define $\varphi$ on $\bigcup_{n} g^{(n)}(\{|z|<r\}$ ). (And by Cartan's theorem, used in $\S \mathrm{V}, \bigcup_{n} g^{(n)}(\{|z|<r\})=\mathbb{C}$ or $\left.\mathbb{C}-\{a\}\right]$ for some $a \in \mathbb{C}$.) This is however not necessarily the case.

It seems that what we have seen raises the question: In the set of entire holomorphic functions $z+\sum_{j \geq 2} a_{j} z^{j}$ which are the ones which have a local 
square root, and which are the ones which, in this set, have a nontrivial local conjugacy class? We have only rather trivial examples.

\section{ANALYTIC Classification}

We have seen precise examples of holomorphic germs at 0 in $\mathbb{C}$ that are formally equivalent but not equivalent via holomorphic change of variable. The holomorphic classification of holomorphic germs $f(z)=z+\cdots$ has been done by Ecalle and Voronin ([6, 24], see also the excellent presentations in [13, 9]). But the invariants that they define seem to be almost impossible to compute, on precise examples.

In this section we give a brief discussion of Voronin's classification of holomorphic germs [24] (see also §IV.2), and indicate how at least a part of his work could be accomplished by a small modification of the "basic scheme". In [24], Voronin considers the class $A_{2}$ of (convergent) holomorphic germs

$$
f(z)=z+\sum_{j=2}^{\infty} a_{j} z^{j}
$$

with $a_{2} \neq 0$.

He associates to each such $f$ a "modulus" and shows that $f, g$ in $A_{2}$ are conjugate if and only if they have the same modulus. We sketch a method of constructing this modulus which is different from that of Voronin. In fact it is due to Fatou [7]. What is shown is that there are two domains $\Omega_{1}$ and $\Omega_{2}$, both with 0 in their boundaries such that $\Omega_{1} \cup \Omega_{2} \cup\{0\}$ is a neighborhood of 0 . Moreover, $f$ is conjugate to $f_{0}(z)=\frac{z}{1-z}$ by means of a function $\varphi_{1}$ and in $\Omega_{2}$ by means of a function $\varphi_{2}$. The intersection of $\Omega_{1}$ with $\Omega_{2}$ consists of the disjoint union of two simply connected domains $\Omega_{+}$and $\Omega_{-}$.

The modulus associated to $f$ consists of the pair of transition functions $\Phi_{ \pm}=\varphi_{2} \circ \varphi_{1}^{-1} \mid \Omega_{ \pm}$(with a simple equivalence relation). Of course, if $f$ is actually conjugate to $f_{0}$ in a neighborhood of 0 then one can take $\varphi_{1}=\varphi_{2}$ and $\Phi_{ \pm}=$id. The existence of the domains $\Omega_{1}, \Omega_{2}$ and the conjugators $\varphi_{1}$, $\varphi_{2}$ is established in [7, $\S 8,9$, and 10, pp. 191-206]. In fact both Fatou and Voronin prefer to make a change of variable that takes 0 to $\infty$. Conjugating by this change of variable takes $f_{0}$ to the translation $z \mapsto z+1$. If $R(z)$ is the function obtained by conjugating our original $f(z)$ then what is required is to find a solution to the functional equation $F(R(z))=F(z)+1$, called Abel's equation.

In the present situation, the "basic scheme" would consist in setting $f(z)=$ $\lim \left(R^{n}(z)-n\right)$. Fatou has to use a second-order perturbation by setting instead $F(z)=\lim \left(R^{n}(z)-n-a \log n\right)$, for an appropriate value of $a$. See [7, pp. 194-197].

\section{TWO REAL VARIABLES}

Diffeomorphism of class $\mathscr{C}^{l}$, or germ of diffeomorphism, of $\left(\mathbb{R}^{2}, 0\right)$, means a map of class $\mathscr{C}^{l}$, defined in some neighborhood of 0 in $\mathbb{R}^{2}$, with values in $\mathbb{R}^{2}$, mapping 0 to 0 , and with a $\mathscr{C}^{l}$ local inverse. Although we have looked for precise statements ( $\mathscr{C}^{3 p}$ regularity) there is no claim that our results are close to being sharp. Before starting our discussion, let us point out that there are real analytic (and even polynomial) germs of diffeomorphisms of $\left(\mathbb{R}^{2}, 0\right)$ 
that are $\mathscr{C}^{\infty}$ but not real analytically conjugate. Such examples derive easily from corresponding examples in one variable. Take $F(x, y)=\left(x+x^{2}, y\right)$ and $G(x, y)=\left(x+x^{2}+\sum_{j \geq 4} a_{j} x^{j}, y\right)$. Then $F$ and $G$ are $\mathscr{C}^{\infty}$ conjugate, but not real analytically conjugate, if the radius of convergence of the series $\sum a_{j} x^{j}$ is $+\infty$, unless $F=G$.

\section{Formal theory in $\mathbb{R}^{2}$.}

Lemma 9. Let $F$ and $G$ be formal power series $F(z)=z+z^{p}+\sum_{j=p+1}^{\infty} a_{j} z^{j}$ $(p \geq 2)$ and $G(z)=z+z^{p}+\sum_{j=p+1}^{\infty} A_{j} z^{j}$. Let $\psi$ be a formal power series $\psi(z, \bar{z})=\sum_{j+k \geq 1} \alpha_{j, k} z^{j} \bar{z}^{k}$ with nonvanishing first-order term (i.e. $\left|\alpha_{1,0}\right|+$ $\left.\left|\alpha_{0,1}\right| \neq 0\right)$. If $F \circ \psi=\psi \circ G$ there exists $r \in\{0, \ldots, p-2\}$ such that

$$
\text { either } \begin{aligned}
\psi & =e^{2 i \pi r /(p-1)} z+\sum_{j \geq 2} \alpha_{j, 0} z^{j} \\
\text { or } \psi & =e^{2 i \pi r /(p-1)} \bar{z}+\sum_{j \geq 2} \alpha_{0, j} \bar{z}^{j} .
\end{aligned}
$$

Proof. The term of degree $p$ in $F \circ \psi-\psi \circ G$ is

$$
\alpha_{1,0}^{p} z^{p}+\alpha_{0,1}^{p} \bar{z}^{p}+\sum_{l=1}^{p-1}\left(\begin{array}{l}
l \\
p
\end{array}\right) \alpha_{1,0}^{p-l} \alpha_{0,1}^{l} z^{p-l} \bar{z}^{l}-\alpha_{1,0} z^{p}-\alpha_{0,1} \bar{z}^{p} .
$$

One must have $\alpha_{1,0} \alpha_{0,1}=0$, and $\alpha_{1,0}^{p}=\alpha_{1,0} \alpha_{0,1}^{p}=\alpha_{0,1}$. So the possible linear parts for $\psi$ are $e^{2 \pi i r /(p-1)} z$ or $e^{2 \pi i r /(p-1)} \bar{z}$ for some $r \in\{0, \ldots, p-1\}$. Replacing $F$ by its conjugate $\left(e^{-2 \pi i r /(p-1)} z\right) \circ F \circ\left(e^{2 \pi i r /(p-1)} z\right)$ one can assume $r=0$. And similarly one can reduce the case $\psi=\bar{z}+\cdots$ to the case $\psi=z+\cdots$ by replacing $F$ by $\bar{z} \circ F \circ \bar{z}$, and $\psi$ by $\bar{\psi}$. So from now on, we will assume that

$$
\psi=z+\sum_{j+k \geq 2} \alpha_{j, k} z^{j} \bar{z}^{k}
$$

We have to show that $\alpha_{j, k}=0$ if $k>0$. Assume that we have already shown that

$$
\psi=z+\sum_{j=2}^{q-1} \alpha_{j, 0} z^{j}+\sum_{j+k \geq q} \alpha_{j, k} z^{j} \bar{z}^{k} \quad(q \geq 2) .
$$

Set

$$
Q_{q}(z)=\sum_{k=1}^{q} \alpha_{q-k, k} z^{q-k} \bar{z}^{k}
$$

The nonholomorphic terms of (total) degree $q+(p-1)$ in $F \circ \psi-\psi \circ G$ are the same as in $\left(z+Q_{q}(z)\right)^{p}-Q_{q}\left(z+z^{p}\right)$ and therefore the same as in $p z^{p-1} Q_{q}(z)-Q_{q}\left(z+z^{p}\right)$. In this difference the coefficient of $\bar{z}^{q+p-1}$ is $-q \alpha_{0, q}$. Therefore $\alpha_{0, q}=0$. Then, in the same way, if we have already shown that $\alpha_{0, q}=\alpha_{1, q-1}=\cdots=\alpha_{q-k, k}=0$ for $k>2$, we see that the coefficient of $z^{q-k+1} \bar{z}^{p+k-2}$ in $F \circ \psi-\psi \circ G$ is $-(k-1) \alpha_{q-k+1, k-1}$. Hence $\alpha_{q-k+1, k-1}=0$. And, by induction, the lemma is proved.

Remark. It is important to notice that in the proof that we have given, the determination of the coefficients of the terms of degree $\leq q$ in $\psi$ depends 
only on consideration of the terms of degree $\leq q+(p-1)$ in $F$ and $G$. This leads to the following:

If $F$ and $G$ are as in Lemma 9, and if $\psi$ is a local diffeomorphism of $\left(\mathbb{R}^{2}, 0\right)$, of class $\mathscr{C}^{q+(p-1)}$ such that $F \circ \psi=\psi \circ G$ then, for some $r \in$ $\{0, \ldots, p-2\}$ :

$$
\begin{aligned}
\text { either } \psi & =e^{2 i \pi r /(p-1)} z+\sum_{j=2}^{q} \alpha_{j, 0} z^{j}+\mathscr{O}\left(|z|^{q+1}\right) \\
\text { or } \psi & =e^{2 i \pi r /(p-1)} \bar{z}+\sum_{j=2}^{q} \alpha_{j, 0} \bar{z}^{j}+\mathscr{O}\left(|z|^{q+1}\right) .
\end{aligned}
$$

In the first case $\bar{\partial} \psi=\mathscr{O}\left(|z|^{q}\right)$; in the second case $\partial \psi=\mathscr{O}\left(|z|^{q}\right)$.

2. Proposition 6, Corollary. In this subsection, we state and prove the facts that allow us to apply the analytic results in the differentiable setting.

Proposition 6. Let $F$ and $G$ be germs of holomorphic functions at 0 in $\mathbb{C}, F(z)$ $=z+a_{p} z^{p}+\sum_{j \geq p+1} a_{j} z^{j} \quad\left(p>1\right.$ and $\left.a_{p} \neq 0\right)$, and $G(z)=z+\cdots$. Let $\psi$ be a local diffeomorphism of $\left(\mathbb{R}^{2}, 0\right)$, of class $\mathscr{C}^{3 p}$. If $F \circ \psi=\psi \circ G$, then $\psi$ is holomorphic or antiholomorphic, near 0 .

After a linear change of coordinates we can assume that $F=z+z^{p}+$ higherorder terms (i.e. $a_{p}=1$ ).

Lemma 10. Let $\varepsilon>0$. There exists $\eta>0$ such that for every $z \in \mathbb{C},|z|<\eta$ the following holds:

(1) If $\operatorname{Re} z^{p-1} \leq\left|\operatorname{Im} z^{p-1}\right|$, for $k>0$ large enough

$$
\left|F^{(k)}(z)\right| \leq(1+\varepsilon)[(p-1) k]^{-1 /(p-1)} .
$$

(2) If $\operatorname{Re} z^{p-1} \geq-\left|\operatorname{Im} z^{p-1}\right|$, for $k<0$, and $|k|$ large enough

$$
\left|F^{(k)}(z)\right| \leq(1+\varepsilon)[(p-1)|k|]^{-1 /(p-1)} .
$$

It is part of this statement that $F^{(k)}$ is defined for all integers $k$ that are positive in the first case, negative in the second case.

Proof. Set $\zeta=\frac{1}{z}$ (a change of variable already used by Fatou). Then $z \mapsto F(z)$ corresponds, via conjugation by $\frac{1}{z}$, to a map $\zeta \mapsto \widetilde{F}(\zeta)$ defined near $\infty$ and $\widetilde{F}(\zeta)=\zeta-\frac{1}{\zeta^{p-2}}+\mathscr{O}\left(\frac{1}{\zeta^{p-1}}\right)$.

The lemma is equivalent to showing that for every $\varepsilon>0$, there exists $R>0$ such that for every $\zeta \in \mathbb{C},|\zeta| \geq 2 R:\left|\widetilde{F}^{(k)}(\zeta)\right| \geq\left[((1-\varepsilon)(p-1)|k|]^{1 /(p-1)}\right.$, for $k>0$ if $\operatorname{Re} \zeta^{p-1} \leq\left|\operatorname{Im} \zeta^{p-1}\right|$, for $k<0$ if $\operatorname{Re} \zeta^{p-1} \geq-\left|\operatorname{Im} \zeta^{p-1}\right|$, and in both cases for $|k|$ large enough, depending possibly on $\zeta$.

(1) Case $p=2$. Then $\widetilde{F}(\zeta)=\zeta-1+\mathscr{O}\left(\frac{1}{\zeta}\right)$. Fix $\varepsilon \in\left(0, \frac{1}{\sqrt{5}}\right)$. Choose $R>0$ so that $|\widetilde{F}(\zeta)-(\zeta-1)| \leq \frac{\varepsilon}{2}$ for $|\zeta| \geq \frac{R}{\sqrt{5}}$.

Notice that the region $\Omega$ defined by

$$
\Omega=\left\{\zeta \in \mathbb{C},|\operatorname{Im} \zeta| \geq \frac{\operatorname{Re} \zeta}{2}+\frac{R}{2}, \operatorname{Re} \zeta \leq|\operatorname{Im} \zeta|\right\}
$$

omits entirely the region $|\zeta|<\frac{R}{\sqrt{5}}$, and is stable under $\widetilde{F}$ (i.e. $\widetilde{F}(\Omega) \subseteq \Omega$ ), due to $\varepsilon<\frac{1}{\sqrt{5}}$. Therefore if $\zeta \in \Omega$, one gets by induction on $k \quad(k>$ 
$0) \operatorname{Re} \widetilde{F}^{(k)}(\zeta) \leq-\left(1-\frac{\varepsilon}{2}\right) k+\operatorname{Re} \zeta$. Since the set of points $\zeta$ such that $\operatorname{Re} \zeta \leq$ $|\operatorname{Im} \zeta|$ and $|\bar{\zeta}|>2 R$ is included in $\Omega$, this establishes the assertion of the lemma for the points $\zeta$ such that $\operatorname{Re} \zeta \leq|\operatorname{Im} \zeta|$ (equivalently $\operatorname{Re} z \leq|\operatorname{Im} z|$ ).

One does similarly for the case $\operatorname{Re} \zeta \geq-|\operatorname{Im} \zeta|$ by letting $k$ tend to $-\infty$. Or, if one prefers, one can reduce to the previous case by replacing $F$ by $-F^{-1}(-z)$.

(2) Case $p>2$. Then $(\widetilde{F}(\zeta))^{p-1}=\zeta^{p-1}-(p-1)+\mathscr{O}\left(\frac{1}{\zeta}\right)$. The arguments used above then lead to

$$
\left|\widetilde{F}^{(k)}(\zeta)\right|^{p-1} \geq(1-\varepsilon)(p-1)|k|,
$$

for $|\zeta| \geq R$, for $k>0$ if $\operatorname{Re} \zeta^{p-1} \leq\left|\operatorname{Im} \zeta^{p-1}\right|$, and for $k<0$ if $\operatorname{Re} \zeta^{p-1} \geq$ $-\left|\operatorname{Im} \zeta^{p-1}\right|$, for $|k|$ large enough.

The lemma being established we now turn to the Proof of Proposition 6.

Proof of Proposition 6. One can replace $G$ by any function which is conjugate to $G$, via some holomorphic change of variable, so one can assume that also $G=z+z^{p}+\cdots$.

By Lemma 9 and the remark following it

$$
\frac{\partial \psi}{\partial \bar{z}}(z)=\mathscr{O}\left(|z|^{2 p+1}\right) \quad \text { or } \quad \frac{\partial \psi}{\partial z}(z)=\mathscr{O}\left(|z|^{2 p+1}\right) .
$$

Replacing $F$ by $\bar{z} \circ F \circ \bar{z}$ if needed, and $\psi$ by $\bar{\psi}$, we can assume that $\frac{\partial \psi}{\partial \bar{z}}=$ $\mathscr{O}\left(|z|^{2 p+1}\right)$. We then have to show that $\psi$ is holomorphic, near 0 .

From $F \circ \psi=\psi \circ G$, one gets that for every $k \in \mathbb{Z}$, near $0, F^{(k)} \circ \psi=$ $\psi \circ G^{(k)}$. By differentiating

$$
\left[\left(F^{(k)}\right)^{\prime}(\psi(z))\right] \bar{\partial} \psi(z)=\bar{\partial} \psi\left(G^{(k)}(z)\right) \overline{\left(G^{(k)}\right)^{\prime}}(z) .
$$

Therefore

$$
|\bar{\partial} \psi(z)|=\left|\bar{\partial} \psi\left(G^{(k)}(z)\right)\right|\left|\frac{\left(G^{(k)}\right)^{\prime}(z)}{\left(F^{(k)}\right)^{\prime}(\psi(z))}\right| .
$$

Since $\psi(z)=e^{2 \pi i r /(p-1)} z+\cdots$, for $|z|$ small, either both $z$ and $\psi(z)$ are in the region $\left\{\operatorname{Re} z^{p-1} \leq\left|\operatorname{Im} z^{p-1}\right|\right\}$ or both $z$ and $\psi(z)$ are in the region $\left\{\operatorname{Re} z^{p-1} \geq-\left|\operatorname{Im} z^{p-1}\right|\right\}$.

So one can apply Lemma 10 simultaneously to $F$ and $G$, at $z$ and $z_{1}=$ $\psi(z)$. In the first case, $z$ being fixed, one can let $k$ tend to $+\infty$. We then have

$$
\left(F^{(k)}\right)^{\prime}\left(z_{1}\right)=F^{\prime}\left(F^{(k-1)}\left(z_{1}\right)\right) F^{\prime}\left(F^{(k-2)}\left(z_{1}\right)\right) \cdots F^{\prime}\left(F\left(z_{1}\right)\right) \cdot F^{\prime}\left(z_{1}\right) .
$$

Since $F(t)=t+t^{p}+\cdots, F^{\prime}(t)=1+p t^{p-1}+\mathscr{O}\left(t^{p}\right)$. Using the estimate of Lemma 10, for $\delta>0$ arbitrary, one gets that for $l$ large enough

$$
\left|1-F^{\prime}\left(F^{(l)}\left(z_{1}\right)\right)\right| \leq(1+\delta) \frac{p}{(p-1) l}
$$

This yields the estimate in the product ( $\delta$ maybe different, but still arbitrary)

$$
\left|\left(F^{(k)}\right)^{\prime}(\psi(z))\right| \geq c k^{-(1+\delta) p /(p-1)},
$$

for some constant $c$ independent of $k$.

Similarly

$$
\left|\left(G^{(k)}\right)^{\prime}(z)\right| \leq c k^{(1+\delta) p /(p-1)} .
$$


Finally

$$
|\bar{\partial} \psi(z)| \leq\left|\bar{\partial} \psi\left(G^{(k)}(z)\right)\right| k^{2(1+\delta) p /(p-1)} \quad(k>0) .
$$

Under our present assumptions

$$
\left|G^{(k)}(z)\right|=\mathscr{O}\left(k^{-1 /(p-1)}\right) \text { and } \bar{\partial} \psi(z)=\mathscr{O}\left(|z|^{2 p+1}\right) .
$$

This leads to $\bar{\partial} \psi(z)=\mathscr{O}\left(k^{2(1+\delta) \frac{p}{p-1}-\frac{2 p+1}{p-1}}\right)$. Taking $\delta$ small enough, so that $2 \delta p-1<0$, and letting $k$ tend to $+\infty$ one sees that $\bar{\partial} \psi(z)=0$, as desired.

The case when both $z$ and $\psi(z)$ belong to the region $\left\{\operatorname{Re} z^{p-1} \leq\left|\operatorname{Im} z^{p-1}\right|\right\}$ can be handled similarly by letting $k$ tend to $-\infty$.

Corollary. Let $F$ be a germ of a holomorphic function at 0 in $\mathbb{C}, F(z)=$ $z+a_{p} z^{p}+\sum_{j=p+1}^{\infty} a_{j} z^{j}, p>1$ and $a_{p} \neq 0$. If $f$ is a local diffeomorphism of $\left(\mathbb{R}^{2}, 0\right)$, of class $\mathscr{C}^{3 p}$, such that, for some integer $k, f^{(k)}=F$, then $f$ is holomorphic, or antiholomorphic near 0 .

The corollary follows immediately from Proposition 6 by taking $G=F$ and $\psi=f$.

3. Proof of Proposition 1. We now prove the following precise version of Proposition 1:

Let $f(z)=z+\cdots$ be an entire function, or more generally a holomorphic function on $\mathbb{C}$ with a countable set of singularities. Then $f$ cannot be the time $t$ map of a $\mathscr{C}^{3 p}$ vector field $X$, defined near 0 in $\mathbb{R}^{2}(=\mathbb{C})$, and $X(0)=0$, unless $f(z)=\frac{z}{1+b z}$ for some $b \in \mathbb{C}$.

Proof. Without loss of generality we can assume that $t=1$. By the corollary to Proposition 6 the map $T_{1 / k} \quad\left(T_{1 / k}^{X}\right.$ to be more precise) must be holomorphic or antiholomorphic near 0 . But since $T_{1 / k}=T_{1 / 2 k} \circ T_{1 / 2 k}, T_{1 / k}$ must in fact be holomorphic near 0 . Next we claim that $T_{1 / k}$ is holomorphic on some neighborhood of 0 which does not shrink as $k$ tends to $\infty$. There seem to be several arguments to get this. Let us just mention the following one: In proving proposition 6, the dynamics which has been used is the one of $F$ and $G$, not the one of $\psi$. Here, we apply the proposition to $F=G=f$, and $\psi=T_{1 / 2 k}$. A uniform control of the, say, $\mathscr{C}^{2}$ norm of $\psi$ is all that is needed. (But the reader may prefer not to look at the proof of Proposition 6, and use again the commutation of $T_{1 / 2 k}$ and $f$.)

Since $X(z)=\lim _{k \rightarrow \infty} k\left(T_{1 / k}(z)-z\right)$, the vector field $X$ should be a holomorphic vector field, which is impossible due to Proposition 1.1.

4. Proof of Proposition 2. We have seen that $z+z^{2}$ has no holomorphic $k$ th root $(k>1)$ in any neighborhood of 0 . And the only entire functions $f$ which are conjugate to $z+z^{2}$, under a local holomorphic change of variable at 0 , are the functions $z+a z^{2}$, for $a \neq 0$.

We now start the proof of Proposition 2.

Let $G(z)=z+\cdots$ the germ at 0 of an entire function. Assume that $G$ is $\mathscr{C}^{6}$ conjugate to $z+z^{2}=F_{0}$, i.e. $F_{0} \circ \psi=\psi \circ G$ for some $\mathscr{C}^{6}$ diffeomorphism of $\left(\mathbb{R}^{2}, 0\right)$. By Proposition $6(p=2) \psi$ must be holomorphic or antiholomorphic, near 0 . If $\psi$ is holomorphic then $G=z+a z^{2}$. If $\psi$ is antiholomorphic, notice that $\bar{z} \circ F_{0} \circ \bar{z}=F_{0}$. So $F_{0} \circ(\bar{z} \circ \psi)=(\bar{z} \circ \psi) \circ G$, and $\bar{z} \circ \psi$ is holomorphic. Therefore, again, $G=z+a z^{2}$. 
Finally we wish to show that $z+z^{2}$ has no $k$ th root $(k>1)$. If $f$ were a $k$ th root of $F_{0}=z+z^{2}$, the corollary to Proposition 6 shows that $f$ should be holomorphic or antiholomorphic, near 0 . It has been shown that $f$ cannot be holomorphic. We have to show that $f$ cannot be antiholomorphic.

Assume that $f$ is an antiholomorphic $k$ th root of $F_{0}$; then $f$ and $F_{0}$ commute. Also $F_{0}$ commutes with $\bar{z}$. Therefore $F_{0}$ commutes with both $\bar{z} \circ f$ and $f \circ \bar{z}$. By Lemma 7 there would exist $n \in \mathbb{N}$ such that $F_{0}^{(n)} \circ(\bar{z} \circ f)$ and $F_{0}^{(n)} \circ(f \circ \bar{z})$ extend to entire functions on $\mathbb{C}$. But

$$
\left(\left[F_{0}^{(n)} \circ(f \circ \bar{z})\right] \circ\left[F_{0}^{(n)} \circ(\bar{z} \circ f)\right]\right)^{(k)}=\left[F_{0}^{(2 n)} \circ f^{(2)}\right]^{(k)}=F_{0}^{(2 k n)} \circ f^{(2 k)}=F_{0}^{(2 k n+2)}
$$

is a polynomial.

The end of the proof is as in $\S$ VI. It follows from the above that $F_{0}^{(n)} \circ f$ must be a (antiholomorphic) polynomial. Let $r$ be its degree. $\left(F_{0}^{(n)} \circ f\right)^{(k)}$ is then of degree $r^{k}$, but on the other hand $\left(F_{0}^{(n)} \circ f\right)^{(k)}=F_{0}^{(k n+1)}$ is of degree $2 \cdot 2^{k n}$ which is not the $k$ th power of an integer. So we reached a contradiction, as desired.

Remark. Let $F=z+z^{2}+a_{3} z^{3}+\cdots$ be a formal power series. Then $F$ has a unique formal square root of holomorphic type $\left(\sum_{j \geq 1}, b_{j} z^{j}\right)$. If $a_{3} \neq \mathbb{R}$, this is the only formal square root of $F$. But if $a_{3} \in \mathbb{R}$, for every $\beta \in \mathbb{R}, F$ has a unique formal square root of antiholomorphic type

$$
\bar{z}+\left(\frac{1}{2}+i \beta\right) \bar{z}^{2}+\sum_{j \geq 3} b_{j} \bar{z}^{j} .
$$

Added in proof. The questions (flows, square roots, etc...), considered in this paper in the setting of $\mathbb{R}, \mathbb{R}^{2}$ or $\mathbb{C}$, can obviously be raised in higher dimensions. We thank the referee for pointing out the work by J. Ecalle L'équation du pont et la classification des objets locaux (Publ. Math. Orsay (1985)) does include a discussion of these topics. See especially pages 288-321.

\section{REFERENCES}

1. I. N. Baker, Zusammensetzungen ganzer Funktionen, Math. Z. 69 (1958), 121-163.

2. $\ldots$ Permutable power series and regular iteration, J. Austral. Math. Soc. 2 (1962), 265-294.

3. - Fractional iteration near a fixpoint of multiplier 1, J. Austral. Math. Soc. 4 (1964), 143-151.

4. $\ldots$, Non embeddable functions with a fixpoint of multiplier 1, Math. Z. 99 (1967), 377-384.

5. H. Cartan, Les fonctions analytiques de deux variables et le problème de la représentation analytique, J. Math. Pures Appl. 10 (1931), 1-14.

6. J. Ecalle, Les fonctions résurgentes et leurs applications, Publ. Math. Orsay.

7. P. Fatou, Sur les equations fonctionnelles, Bull. Soc. Math. France 47 (1919), 161-271.

8. M. W. Hirsch and S. Smale, Differential equations, and liner algebra, Academic Press, 1974.

9. Y. S. Il'yashenko, Nonlinear Stokes phenomena, Adv. in Soviet Math., vol. 14, Amer. Math. Soc., Providence, RI, 1993.

10. S. Kobayaski, Hyperbolic manifolds and holomorphic mappings, Marcel Dekker, New York, 1970. 
11. N. Kopell, Commuting diffeomorphisms, Global Analysis, Proc. Sympos. Pure Math., vol. 14, Amer. Math. Soc., Providence, RI, 1970, pp. 165-185.

12. L. S. O. Liverpool, Fractional iteration near a fixed point of multiplier 1, J. London Math. Soc. (2) 9 (1975), 599-609.

13. B. Malgrange,Travaux d'Ecalle et de Martinet-Ramis sur les systèmes dynamiques, Séminaire, Bourbaki no. 582, 1981/82.

14. J. Robbin, Unfolding of discrete dynamical systems, Ergodic Theory Dynamical Systems 4 (1984), 421-486.

15. J. P. Rosay and W. Rudin, Holomorphic maps from $\mathbb{C}^{n}$ to $\mathbb{C}^{n}$, Trans. Amer. Math. Soc. 310 (1988), 47-86.

16. S. Saks and A. Zygmund, Analytic functions, 3rd ed., PWN, Warsaw, 1971.

17. F. Sergeraert, Feuilletages et difféomorphismes infiniment tangents à l'identité, Invent. Math. 39 (1977), 253-275.

18. C. L. Siegel, Iteration of analytic functions, Ann. of Math. (2) 43 (1942), 607-612.

19. S. Sternberg, Local $C^{n}$ transformations of the real line, Duke Math. J. 24 (1957), 97-102.

20. L_ Local contractions and a theorem of Poincaré, Amer. Math. J. 79 (1957), 809-824.

21. G. Szekeres, Regular iteration of real and complex functions, Acta Math. 100 (1958), 203258.

22. - Fractional iteration of entire and rational functions, J. Austral. Math. Soc. 4 (1964), 129-142.

23. F. Takens, Normal forms for certain singularities of vector fields, Ann. Inst. Fourier (Grenoble) 23 (1973), 163-195.

24. S. M. Voronin, Analytic classification of germs of conformal mappings $(\mathbb{C}, 0) \rightarrow(\mathbb{C}, 0)$ with identity linear part, Funktsional. Anal. i Prilozhen. 15 (1981), no. 1, 1-17.

Department of Mathematics, University of Wisconsin, Madison, Wisconsin 53706 